\title{
The Nanograv Nine-Year Data Set: Measurement And Analysis Of Variations In Dispersion Measures
}

\author{
M.L. Jones \\ M.A. McLaughlin \\ J.M. Cordes
}

M. T.Lam

Follow this and additional works at: https://researchrepository.wvu.edu/faculty_publications

\section{Digital Commons Citation}

Jones, M. L.; McLaughlin, M. A.; Cordes, J. M.; and Lam, M. T., "The Nanograv Nine-Year Data Set: Measurement And Analysis Of Variations In Dispersion Measures" (2017). Faculty Scholarship. 964.

https://researchrepository.wvu.edu/faculty_publications/964 


\title{
The NANOGrav Nine-year Data Set: Measurement and Analysis of Variations in Dispersion Measures
}

\author{
M. L. Jones ${ }^{1,2,20}$, M. A. McLaughlin ${ }^{1,2}$, M. T. Lam ${ }^{1,2}$, J. M. Cordes ${ }^{3}$, L. Levin ${ }^{4}$, S. Chatterjee ${ }^{3}$, Z. Arzoumanian ${ }^{5}$, K. Crowter ${ }^{6}$, \\ P. B. Demorest ${ }^{7}$, T. Dolch $^{8}$, J. A Ellis ${ }^{9,21}$, R. D. Ferdman ${ }^{10,11}$, E. Fonseca ${ }^{10}$, M. E. Gonzalez ${ }^{6,12}$, G. Jones ${ }^{13}$, T. J. W. Lazio ${ }^{9}$, \\ D. J. Nice ${ }^{14}$, T. T. Pennucci ${ }^{13}$, S. M. Ransom ${ }^{15}$, D. R. Stinebring ${ }^{16}$, I. H. Stairs ${ }^{6}$, K. Stovall ${ }^{7,17}$, J. K. Swiggum ${ }^{18}$, and W. W. Zhu ${ }^{19}$ \\ ${ }^{1}$ Department of Physics and Astronomy, West Virginia University, Morgantown, WV 26506, USA \\ ${ }^{2}$ Center for Gravitational Waves and Cosmology, West Virginia University, Chestnut Ridge Research Building, Morgantown, WV 26505, USA \\ ${ }^{3}$ Department of Astronomy and Cornell Center for Astrophysics and Planetary Science, Cornell University, Ithaca, NY 14853, USA \\ 4 Jodrell Bank Centre for Astrophysics, Alan Turing Building, School of Physics and Astronomy, The University of Manchester, Oxford Road, Manchester, M13 9PL, UK \\ ${ }^{5}$ Center for Research and Exploration in Space Science and Technology and X-Ray Astrophysics Laboratory, NASA Goddard Space Flight Center, Code 662, \\ Greenbelt, MD 20771, USA \\ ${ }^{6}$ Department of Physics and Astronomy, University of British Columbia, 6224 Agricultural Road, Vancouver, BC V6T 1Z1, Canada \\ ${ }^{7}$ National Radio Astronomy Observatory, P.O. Box 0, Socorro, NM 87801, USA \\ ${ }^{8}$ Department of Physics, Hillsdale College, 33 E. College Street, Hillsdale, MI 49242, USA \\ 9 Jet Propulsion Laboratory, California Institute of Technology, 4800 Oak Grove Dr. Pasadena, CA 91109, USA \\ ${ }^{10}$ Department of Physics, McGill University, 3600 rue Universite, Montreal, QC H3A 2T8, Canada \\ ${ }^{11}$ Department of Physics, University of East Anglia, Norwich NR4 7TJ, UK \\ ${ }^{12}$ Department of Nuclear Medicine, Vancouver Coastal Health Authority, Vancouver, BC V5Z 1M9, Canada \\ ${ }^{13}$ Department of Physics, Columbia University, 550 W. 120th St. New York, NY 10027, USA \\ ${ }^{14}$ Department of Physics, Lafayette College, Easton, PA 18042, USA \\ ${ }^{15}$ National Radio Astronomy Observatory, 520 Edgemont Road, Charlottesville, VA 22903, USA \\ ${ }^{16}$ Department of Physics \& Astronomy, Oberlin College, Oberlin, OH 44074, USA \\ ${ }^{17}$ Department of Physics and Astronomy, University of New Mexico, Albuquerque, NM 87131, USA \\ ${ }^{18}$ Center for Gravitation, Cosmology and Astrophysics, Department of Physics, University of Wisconsin-Milwaukee, P.O. Box 413, Milwaukee, WI 53201, USA \\ ${ }^{19}$ Max-Planck-Institut für Radioastronomie, Auf dem Hügel 69, D-53121, Bonn, Germany \\ Received 2016 December 8; revised 2017 May 15; accepted 2017 May 15; published 2017 June 5
}

\begin{abstract}
We analyze dispersion measure (DM) variations of 37 millisecond pulsars in the nine-year North American Nanohertz Observatory for Gravitational Waves (NANOGrav) data release and constrain the sources of these variations. DM variations can result from a changing distance between Earth and the pulsar, inhomogeneities in the interstellar medium, and solar effects. Variations are significant for nearly all pulsars, with characteristic timescales comparable to or even shorter than the average spacing between observations. Five pulsars have periodic annual variations, 14 pulsars have monotonically increasing or decreasing trends, and 14 pulsars show both effects. Of the four pulsars with linear trends that have line-of-sight velocity measurements, three are consistent with a changing distance and require an overdensity of free electrons local to the pulsar. Several pulsars show correlations between DM excesses and lines of sight that pass close to the Sun. Mapping of the DM variations as a function of the pulsar trajectory can identify localized interstellar medium features and, in one case, an upper limit to the size of the dispersing region of 4 au. Four pulsars show roughly Kolmogorov structure functions (SFs), and another four show SFs less steep than Kolmogorov. One pulsar has too large an uncertainty to allow comparisons. We discuss explanations for apparent departures from a Kolmogorov-like spectrum, and we show that the presence of other trends and localized features or gradients in the interstellar medium is the most likely cause.
\end{abstract}

Key words: ISM: general - pulsars: general

\section{Introduction}

The principal goal of the North American Nanohertz Observatory for Gravitational Waves (NANOGrav; McLaughlin 2013) is to detect gravitational waves in the nanohertz regime of the gravitational wave spectrum using a pulsar timing array (PTA). Sensitivity improves as more millisecond pulsars (MSPs) are added to the PTA, and therefore it is essential to have as many well-timed MSPs as possible (Siemens et al. 2013; Vigeland \& Siemens 2016). For every MSP, we must construct an accurate timing model that accounts for all known effects on the pulsar times of arrival (TOAs) over decade timescales (Jenet et al. 2005; Cordes \& Shannon 2010). One of the parameters that must be fit in the timing

\footnotetext{
${ }^{20}$ STEM Mountains of Excellence Fellow.

21 Einstein Fellow.
}

model is the dispersion measure (DM). As the pulsar signal travels through the interstellar medium (ISM), it encounters ionized plasma and electron density variations along the way. The DM is the integrated column density of free electrons along the line of sight (LOS) to a pulsar:

$$
\mathrm{DM}=\int_{0}^{d} n_{e}(l) d l,
$$

where $n_{e}$ is the free electron density along a $\operatorname{LOS} l$, and $d$ is the pulsar distance. When the pulsar signal propagates through the ISM, interactions with these free electrons cause dispersion that is characterized by a frequency-dependent time delay:

$$
\Delta t \simeq 4.15 \times 10^{6} \mathrm{~ms} \times \mathrm{DM}\left(\frac{1}{\nu_{1}^{2}}-\frac{1}{\nu_{2}^{2}}\right),
$$


where $\nu_{1}$ and $\nu_{2}$ are two different frequencies in $\mathrm{MHz}$, and DM is in $\mathrm{pc} \mathrm{cm}^{-3}$. Observing at least two frequencies is necessary to solve for the DM for a measured time delay. This time delay can be significant when compared to the pulsar period, and therefore the DM must be fit when creating a timing model and corrected for at each epoch (e.g., Demorest et al. 2013; Arzoumanian et al. 2015).

Inhomogeneities in the ISM and solar wind and differences in the relative velocity of the pulsar and the Earth can change the free electron density along the LOS (Lam et al. 2016). The result is a DM that varies with time, changing on timescales of hours to years. In this paper we discuss the variations seen in the NANOGrav nine-year data release (Arzoumanian et al. 2015), constrain the possible sources of these variations, and use these constraints to characterize the ISM along the LOS.

In Section 2, we discuss the data used for this analysis. In Section 3, we discuss the significance and trends seen in the DM time series. In Section 4, we perform a structure function (SF) analysis on select MSPs and put the results in the context of a Kolmogorov spectrum. In Section 5, we discuss the results of these analyses, and in Section 6 we present conclusions.

\section{Data}

Our analysis uses data from the NANOGrav nine-year data set (Arzoumanian et al. 2015). Pulsars were included in the data set based on the anticipated stability of their timing, their TOA precision, and their detection over a wide frequency range. Of the 37 MSPs included in the data release, 17 were reported on in Demorest et al. (2013). Observations took place roughly once a month between 2004 and 2013 with observing time spans of individual pulsars ranging from 0.6 to 9.0 years. Those MSPs with declinations between $0^{\circ}$ and $39^{\circ}$ were observed with the $305 \mathrm{~m}$ William E. Gordon Telescope at the Arecibo Observatory, and the rest were observed with the $100 \mathrm{~m}$ Robert C. Byrd Green Bank Telescope (GBT) of the National Radio Astronomy Observatory (NRAO); PSRs J1713+0747 and B1937+21 were observed with both telescopes. Every MSP was observed at multiple frequencies to account for frequencydependent dispersion effects. Dual-frequency observations occurred within $\sim 1 \mathrm{hr}$ at Arecibo and within several days at the GBT. The typical length of an observation was $\sim 25$ minutes. A more detailed and thorough description of these observations can be found in Arzoumanian et al. (2015).

For each pulsar, the DM was measured at nearly every observing epoch and recorded using the DMX parameter as part of the TEMPO software package, where DMX models DM as constant over a chosen time window (14 days in this case). The $\triangle \mathrm{DM}$, an offset from a globally fixed fiducial DM, is fit as a free parameter in the timing model. The possible errors in $\mathrm{DM}(t)$ estimation using this method are discussed in Lam et al. (2015). Errors on DM are $1 \sigma$ and are determined from the timingparameter covariance matrix after the least-squares timing model fit. Data from early single-receiver observations were omitted for PSRs J1741+1351, J1853+1303, J1910+1256, J1944+0907, and B1953+29 as it was not possible to independently measure DM and other timing properties. We plot DMs versus time (i.e., $\mathrm{DM}(t))$ for all of the pulsars in Figures 1 through 5. Values from the nine-year data release used in this analysis can be seen in Table 1. Partial DM $(t)$ data spans have already been published for 15 pulsars (PSRs J0340+4130, J0613-0200, J1614-2230, $\mathrm{J} 1713+0747$, J1738+0333, J1741+1351, J1744-1134, B1855
+09, J1909-3744, J1910+1256, J1918-0642, B1937+21, $\mathrm{J} 1944+0907$, J2010-1323, and J2302+4442) in Levin et al. (2016).

\section{Determining Significance and Trends in the Variations}

Variability in the DM time series can be seen for many pulsars; it has been a long known effect. The first detection of temporal variations was for the Crab pulsar (Rankin \& Roberts 1971). These variations were later determined to be most likely due to variations in the surrounding nebula (Isaacman \& Rankin 1977). The Vela pulsar exhibits a decreasing time-dependent DM attributed to the pulsar motion through the enveloping supernova remnant (Hamilton et al. 1985).

We first determine whether the DM variations we see are significant or if they are consistent (within errors) with a constant DM value. We calculate the reduced $\chi^{2}$ for each pulsar as

$$
\chi_{r}^{2}=\frac{1}{N_{\text {dof }}} \sum \frac{(\mathrm{DM}(t)-\overline{\mathrm{DM}})^{2}}{\sigma(t)^{2}},
$$

where $N_{\text {dof }}$ is the number of degrees of freedom, $\overline{\mathrm{DM}}$ is the average DM value for the data span for a pulsar (in this model), and $\sigma(t)$ is the error associated with each $\mathrm{DM}(t)$ value. A large $\chi_{r}^{2}$ value then signifies that the DM variations are much greater than the measurement error and are significant when compared to an unchanging fiducial DM. All but two of the pulsars (PSRs $\mathrm{J} 1923+2515$ and $\mathrm{J} 2214+3000$ ) have $\chi_{r}^{2} \geqslant 1$. Of these, we identify 15 pulsars as showing moderate variations (those with $\left.1 \leqslant \chi_{r}^{2} \leqslant 10\right)$, and 20 pulsars with significant variations $\left(\chi_{r}^{2} \geqslant 10\right)$. We therefore conclude that the DMs are intrinsically variable for all of the MSPs in our sample, with the possible exceptions of $\mathrm{J} 1923+2515$ and $\mathrm{J} 2214+3000$, which both show visible variation at a low level despite the statistical test. Both of these pulsars have short data sets (2.2 and 2.1 years, respectively).

\subsection{Systematic Variations}

DMs can vary in many ways, with components that appear linear, periodic, or random. Here we consider systematic DM variations such as linear trends and periodicities. Stochastic contributions are discussed in Section 4. Sources of linear trends and periodicities include a changing distance between the Earth and the pulsar, a wedge with linear density changes in the ISM or the orbital motion of the Earth, among others; the possible geometries from which these trends arise are explained in detail in Lam et al. (2016). Both linear and periodic trends have been seen in Parkes Pulsar Timing Array (PPTA) data (You et al. 2007; Keith et al. 2013; Petroff et al. 2013; Reardon et al. 2016). Petroff et al. (2013) determined the significance of a linear trend by calculating the error of a fit to the slope; linear trends were deemed significant if the errors are less than $35 \%$ of the slope value and highly significant if the errors on the slope measurement are less than $20 \%$. This method is not applicable for the NANOGrav nine-year data set as a large number of pulsars exhibit sinusoidal trends without linear trends.

\footnotetext{
${ }^{22}$ TEMPO software package: http://tempo.sourceforge.net.
} 

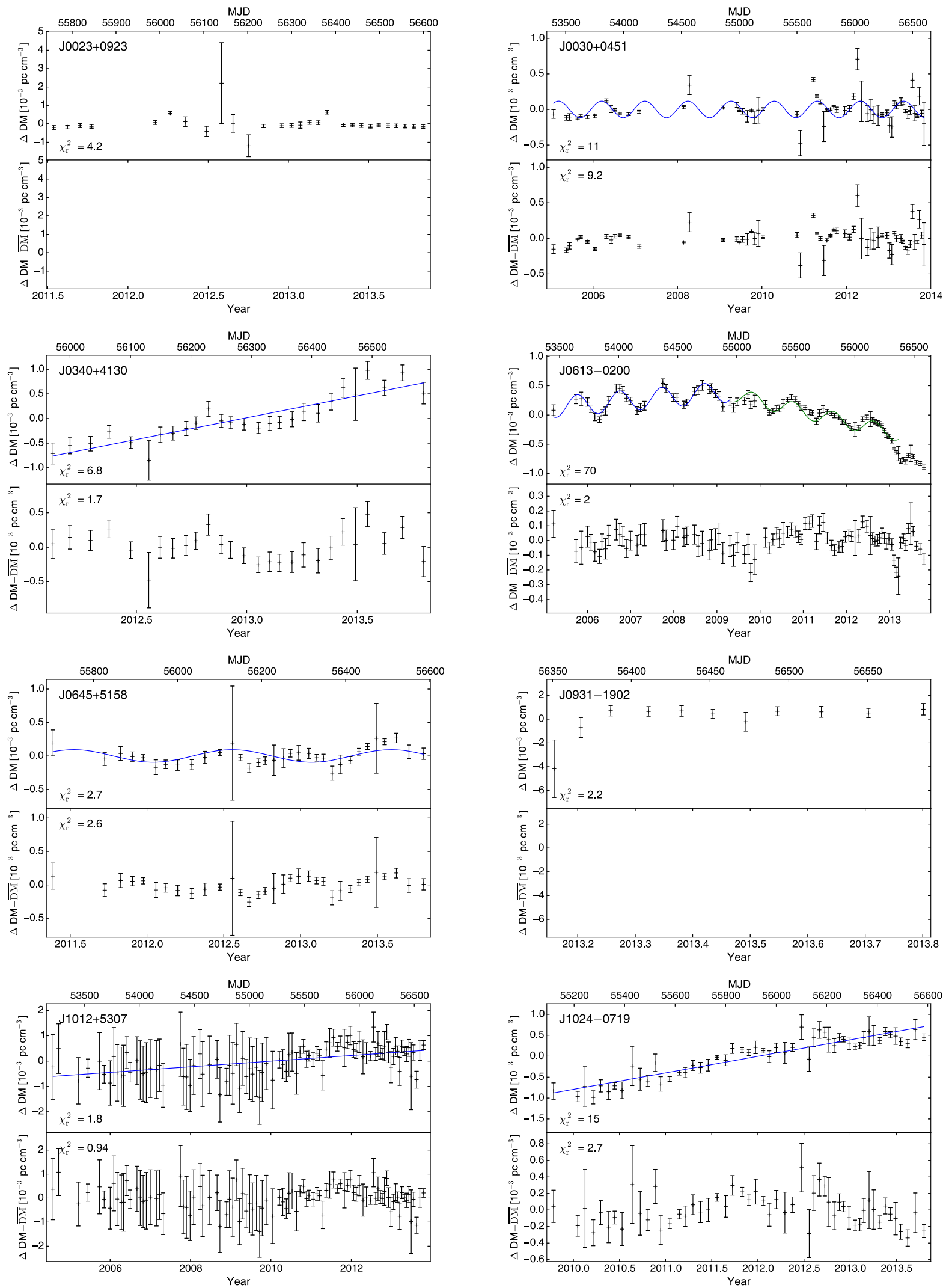

Figure 1. The top panel shows the DM time series with the best-fit function (if applicable) in blue. The zero point for the DM variations corresponds to the fiducial DM for the data span. The error bars are $\pm 1 \sigma$ errors returned by TEMPO. The bottom panel shows the DM residuals after the trend has been removed from the time series; an empty panel means no trend was found. The $\chi_{r}^{2}$ values before and after these fits for each pulsar appear in the top and bottom panels, respectively, as well as in Tables 1 and 2. PSR J0931-1902 has too short a data span for a trend to be determined. Partial DM(t) ranges were published in Levin et al. (2016) for PSRs J0340 +4130 and J0613-0200. 
Table 1

Properties of NANOGrav MSPs in the Nine-year Data Release

\begin{tabular}{|c|c|c|c|c|c|c|c|c|c|}
\hline PSR & $\begin{array}{c}\mu_{\lambda} \\
\left(\operatorname{mas~yr}^{-1}\right)\end{array}$ & $\begin{array}{c}\mu_{\beta} \\
\left(\operatorname{mas~yr}^{-1}\right)\end{array}$ & $\begin{array}{c}\mu_{\alpha} \\
\left(\operatorname{mas~yr}^{-1}\right)\end{array}$ & $\begin{array}{c}\mu_{\delta} \\
\left(\operatorname{mas~yr}^{-1}\right)\end{array}$ & $\begin{array}{c}\mathrm{DM} \\
\left(\mathrm{pc} \mathrm{cm} \mathrm{cm}^{-3}\right)\end{array}$ & $\begin{array}{c}d_{\mathrm{DM}} \\
(\mathrm{kpc})\end{array}$ & $\begin{array}{c}d_{\mathrm{PX}} \\
(\mathrm{kpc})\end{array}$ & $\chi_{r}^{2}$ & $\begin{array}{c}v_{T} \\
\left(\mathrm{~km} \mathrm{~s}^{-1}\right)\end{array}$ \\
\hline $\mathrm{J} 0023+0923$ & $-13.9(2)$ & $-1(1)$ & $-12.3(6)$ & $-6.7(9)$ & 14.3 & $0.7(2)$ & $\cdots$ & 4.2 & $46(13)$ \\
\hline $\mathrm{J} 0030+0451$ & $-5.52(1)$ & $3.0(5)$ & $-6.3(2)$ & $0.6(5)$ & 4.3 & $0.3(1)$ & $0.30(2)$ & 11 & $8.9(7)$ \\
\hline $\mathrm{J} 0340+4130$ & $-2.4(8)$ & $-4(1)$ & $-1.3(7)$ & $-5(1)$ & 49.6 & $1.7(4)$ & $\ldots$ & 6.8 & $38(12)$ \\
\hline J0613-0200 & $2.12(2)$ & $-10.34(4)$ & $1.85(2)$ & $-10.39(4)$ & 38.8 & $1.7(4)$ & $1.1(2)$ & 70 & $55(10)$ \\
\hline $\mathrm{J} 0645+5158$ & $2.1(1)$ & $-7.3(2)$ & $1.4(1)$ & $-7.5(2)$ & 18.2 & $0.7(2)$ & $0.8(3)$ & 2.7 & $29(11)$ \\
\hline J0931-1902 & $\ldots$ & $\ldots$ & $\ldots$ & $\ldots$ & 41.5 & $1.8(5)$ & $\cdots$ & 2.2 & $\ldots$ \\
\hline $\mathrm{J} 1012+5307$ & $13.9(1)$ & $-21.7(3)$ & $2.5(2)$ & $-25.6(2)$ & 9.0 & $0.4(1)$ & $\cdots$ & 1.8 & $49(12)$ \\
\hline J1024-0719 & $-14.36(6)$ & $-57.8(3)$ & $-35.2(1)$ & $-48.0(2)$ & 6.5 & $0.4(1)$ & $\ldots$ & 15 & $113(28)$ \\
\hline J1455-3330 & $8.16(7)$ & $0.5(3)$ & 7.9(1) & $-2.0(3)$ & 13.6 & $0.5(1)$ & $\cdots$ & 2.4 & 19(4) \\
\hline J1600-3053 & $0.47(2)$ & $-7.0(1)$ & $-0.95(3)$ & $-7.0(1)$ & 52.3 & $1.6(4)$ & $3.0(8)$ & 42 & $100(27)$ \\
\hline J1614-2230 & $9.46(2)$ & $-31(1)$ & $3.8(2)$ & $-32(1)$ & 34.5 & $1.3(3)$ & $0.65(5)$ & 20 & $100(8)$ \\
\hline $\mathrm{J} 1640+2224$ & $4.20(1)$ & $-10.73(2)$ & $2.09(1)$ & $-11.33(2)$ & 18.5 & $1.2(3)$ & $\cdots$ & 295 & $66(16)$ \\
\hline J1643-1224 & $5.56(8)$ & $5.3(5)$ & $6.2(1)$ & $4.5(5)$ & 62.4 & $2.3(6)$ & $\cdots$ & 112 & $84(22)$ \\
\hline $\mathrm{J} 1713+0747$ & $5.260(2)$ & $-3.442(5)$ & $4.918(2)$ & $-3.914(5)$ & 16.0 & $0.9(2)$ & $1.18(4)$ & 29 & $35(1)$ \\
\hline $\mathrm{J} 1738+0333$ & $6.6(2)$ & $6.0(4)$ & $6.9(2)$ & $5.8(4)$ & 33.8 & $1.4(4)$ & $\cdots$ & 5.0 & $59(17)$ \\
\hline $\mathrm{J} 1741+1351$ & $-8.8(1)$ & $-7.6(2)$ & $-9.1(1)$ & $-7.2(2)$ & 24.2 & $0.9(2)$ & $\ldots$ & 4.7 & $50(11)$ \\
\hline J1744-1134 & $19.01(2)$ & $-8.68(8)$ & $18.76(2)$ & $-9.20(8)$ & 3.1 & $0.4(1)$ & $0.41(2)$ & 17 & $41(2)$ \\
\hline J1747-4036 & $0.1(8)$ & $-6(1)$ & $0(1)$ & $-6(1)$ & 153.0 & $3.3(8)$ & $\ldots$ & 133 & $\ldots$ \\
\hline J1832-0836 & $\ldots$ & $\ldots$ & $\ldots$ & $\ldots$ & 28.2 & $1.1(3)$ & $\cdots$ & 16 & $\cdots$ \\
\hline $\mathrm{J} 1853+1303$ & $-1.8(2)$ & $-2.9(4)$ & $-1.48(2)$ & $-3.1(4)$ & 30.6 & $2.0(5)$ & $\cdots$ & 5.8 & $32(9)$ \\
\hline B1855+09 & $-3.27(1)$ & $-5.10(3)$ & $-2.651(15)$ & $-5.45(3)$ & 13.3 & $1.2(3)$ & $\cdots$ & 1335 & $34(9)$ \\
\hline $\mathrm{J} 1903+0327$ & $-3.5(3)$ & $-6.2(9)$ & $-2.7(3)$ & $-6.5(9)$ & 297.6 & $6(2)$ & $\ldots$ & 27 & 202(71) \\
\hline J1909-3744 & $-13.868(4)$ & $-34.34(2)$ & $-9.518(4)$ & $-35.79(2)$ & 10.4 & $0.5(1)$ & $1.07(4)$ & 1375 & $188(7)$ \\
\hline $\mathrm{J} 1910+1256$ & $-0.7(1)$ & $-7.2(2)$ & $0.3(1)$ & $-7.2(2)$ & 38.1 & $2.3(6)$ & $\ldots$ & 2.7 & $79(21)$ \\
\hline J1918-0642 & $-7.93(2)$ & $-4.85(9)$ & $-7.18(3)$ & $-5.90(9)$ & 26.6 & $1.2(3)$ & $0.9(2)$ & 171 & $40(9)$ \\
\hline $\mathrm{J} 1923+2515$ & $-9.5(2)$ & $-12.8(5)$ & $-6.6(2)$ & $-14.5(5)$ & 18.9 & $1.6(4)$ & $\ldots$ & 0.9 & $121(30)$ \\
\hline B1937+21 & $-0.02(1)$ & $-0.41(2)$ & $0.07(1)$ & $-0.40(2)$ & 71.0 & $3.6(7)$ & $\cdots$ & 1162 & $7(1)$ \\
\hline $\mathrm{J} 1944+0907$ & $9.4(1)$ & $-25.5(4)$ & $14.37(11)$ & $-23.1(4)$ & 24.3 & $1.8(5)$ & $\ldots$ & 147 & $232(64)$ \\
\hline $\mathrm{J} 1949+3106$ & $13(15)$ & $10(13)$ & $10(11)$ & $13(16)$ & 164.1 & $3.6(9)$ & $\ldots$ & 1.4 & $\ldots$ \\
\hline B1953+29 & $-1.8(9)$ & $-4.4(14)$ & $-0.4(12)$ & $-5(1)$ & 104.5 & $5(1)$ & $\cdots$ & 6.6 & $113(24)$ \\
\hline J2010-1323 & $1.16(4)$ & $-7.3(4)$ & 2.71(9) & $-6.9(4)$ & 22.2 & $1.0(3)$ & $\cdots$ & 70 & $35(11)$ \\
\hline $\mathrm{J} 2017+0603$ & $2.3(6)$ & $-0.1(7)$ & $2.2(7)$ & $0.5(6)$ & 23.9 & $1.6(4)$ & $\cdots$ & 2.8 & $\ldots$ \\
\hline $\mathrm{J} 2043+1711$ & $-8.97(7)$ & $-8.5(1)$ & $-5.85(7)$ & $-10.9(1)$ & 20.7 & $1.7(4)$ & $1.3(4)$ & 6.3 & $100(23)$ \\
\hline $\mathrm{J} 2145-0750$ & $-12.04(4)$ & $-3.7(4)$ & $-10.1(1)$ & $-7.5(4)$ & 9.0 & $0.6(2)$ & $0.8(2)$ & 24 & $48(12)$ \\
\hline $\mathrm{J} 2214+3000$ & 17.1(5) & $-10.5(9)$ & $20.0(6)$ & $-1.7(8)$ & 22.6 & $1.5(4)$ & $\ldots$ & 1.0 & $143(38)$ \\
\hline $\mathrm{J} 2302+4442$ & $-3.3(6)$ & $-1(2)$ & $-2(1)$ & $-3(2)$ & 13.7 & $1.1(3)$ & $\ldots$ & 1.5 & $\ldots$ \\
\hline $\mathrm{J} 2317+1439$ & $0.19(2)$ & $3.80(7)$ & $-1.39(3)$ & $3.55(6)$ & 21.9 & $0.8(2)$ & $1.3(4)$ & 18732 & $14(4)$ \\
\hline
\end{tabular}

Notes. Columns are pulsar name, ecliptic proper motion (longitude and latitude), proper motion in R.A. and decl., the DM, the DM-derived distance, the parallaxderived distance, the reduced chi-squared of the DM time series prior to any fitting, and the transverse velocity. The ecliptic proper motions and DMs were calculated for the nine-year data release (Arzoumanian et al. 2015). Proper motion in R.A. and decl. as well as parallax distances were calculated through timing observations and discussed in Matthews et al. (2016). Proper motion values that are smaller than their uncertainties were not used in subsequent analysis. The DM-derived distances were calculated from the NE2001 model assuming 20\% error (Cordes \& Lazio 2002). The value for $\chi_{r}^{2}$ was calculated using Equation (3). Dashes indicate that no significant measurement was possible. The transverse velocity $v_{T}$ was calculated from the proper motion and the distance estimate with the smaller error (i.e., $d_{\text {DM }}$ or $\left.d_{\mathrm{PX}}\right)$.

In order to determine the scale and structure of the variations, the options being linear, periodic, both, or variations consistent with stochastic noise, we applied a nonlinear least-squares fit to the data using three functions:

$$
\begin{aligned}
& \overline{\mathrm{DM}_{1}}(t)=m t+b, \\
& \overline{\overline{D M}_{2}}(t)=A \cos (\omega t+\phi)+b, \\
& \overline{\overline{D M}_{3}}(t)=A \cos (\omega t+\phi)+m t+b,
\end{aligned}
$$

with the $\chi_{r}^{2}$ calculated for the time series after each of these fits was individually subtracted off. For each fit, $N_{\text {dof }} \approx N-N_{p}$, where $N$ is the number of DM measurements and $N_{p}$ is the number of free parameters being fit in that function $\left(N_{p}=1\right.$ when $\overline{\mathrm{DM}}(t)$ is a constant value). The three $\chi_{r}^{2}$ values were then compared to the original value; the result producing the lowest $\chi_{r}^{2}$ was assigned as the trend. The results of these fits can be seen in Table 2. There is a known complication when 
Table 2

Fitted Trends in the DM Time Series for MSPs in the Nine-year Release

\begin{tabular}{|c|c|c|c|c|c|c|c|c|c|}
\hline PSR & Trend & $\begin{array}{c}d \mathrm{DM} / d t \\
\left(10^{-3} \mathrm{pc} \mathrm{cm}^{-3} \mathrm{yr}^{-1}\right)\end{array}$ & $\begin{array}{c}\text { Amplitude } \\
\left(10^{-4} \mathrm{pc} \mathrm{cm}^{-3}\right)\end{array}$ & $\begin{array}{l}\text { Period } \\
\text { (days) }\end{array}$ & $\chi_{r}^{2}$ & $\begin{array}{c}P_{\mathrm{LS}} \\
\text { (days) }\end{array}$ & $\begin{array}{l}\text { FAP } \\
(\%)\end{array}$ & $\begin{array}{c}\text { Length } \\
\text { (days) }\end{array}$ & $\begin{array}{c}\delta t \\
\text { (days) }\end{array}$ \\
\hline $\mathrm{J} 0023+0923$ & None & $\cdots$ & $\cdots$ & $\cdots$ & $\cdots$ & $\cdots$ & $\cdots$ & 841 & $\cdots$ \\
\hline $\mathrm{J} 0030+0451$ & Periodic & $\cdots$ & $1.2(3)$ & $373(5)$ & 9.2 & 371 & 6.3 & 3204 & 33 \\
\hline $\mathrm{J} 0340+4130$ & Linear & $0.88(9)$ & $\cdots$ & $\cdots$ & 1.7 & 241 & 6.3 & 613 & 73 \\
\hline $\mathrm{J} 0645+5158$ & Periodic & $\cdots$ & $0.9(3)$ & $377(29)$ & 2.6 & 199 & 0.54 & 881 & 78 \\
\hline J0931-1902 & None & $\cdots$ & $\cdots$ & $\cdots$ & $\cdots$ & $\cdots$ & $\cdots$ & 235 & $\cdots$ \\
\hline $\mathrm{J} 1012+5307$ & Linear & $0.11(2)$ & $\cdots$ & $\cdots$ & 0.94 & $\cdots$ & $\cdots$ & 3368 & 2286 \\
\hline J1024-0719 & Linear & $0.39(2)$ & $\cdots$ & $\cdots$ & 2.7 & $\cdots$ & $\cdots$ & 1467 & 148 \\
\hline J1455-3330 & Linear & $0.15(2)$ & $\cdots$ & $\cdots$ & 1.0 & 361 & 7.3 & 3368 & 904 \\
\hline J1614-2230 & Periodic & $\cdots$ & $3.1(5)$ & $370(9)$ & 11 & 370 & 0.17 & 1860 & 14 \\
\hline J1643-1224 & Both & $-1.02(3)$ & $8(1)$ & 387(4) & 10 & 387 & 0.01 & 3293 & 104 \\
\hline $\mathrm{J} 1738+0333$ & Linear & $-0.8(2)$ & $\ldots$ & $\ldots$ & 1.4 & $\ldots$ & $\ldots$ & 1456 & 213 \\
\hline $\mathrm{J} 1741+1351$ & Linear & $-0.12(4)$ & $\cdots$ & $\cdots$ & 1.9 & $\cdots$ & $\cdots$ & 1224 & 287 \\
\hline J1744-1134 & Both & $-0.069(7)$ & $0.4(2)$ & $383(16)$ & 8.0 & $\cdots$ & $\cdots$ & 3369 & 66 \\
\hline J1747-4036 & Linear & $-7.3(4)$ & $\cdots$ & $\cdots$ & 10.0 & 459 & 6.7 & 608 & 16 \\
\hline J1832-0836 & None & $\cdots$ & $\cdots$ & $\cdots$ & $\cdots$ & $\cdots$ & $\cdots$ & 231 & $\cdots$ \\
\hline $\mathrm{J} 1853+1303$ & Linear & $0.12(9)$ & $\cdots$ & $\ldots$ & 5.2 & $\ldots$ & $\ldots$ & 1468 & 361 \\
\hline B1855+09 & Both & $0.382(7)$ & $0.5(3)$ & $364(11)$ & 15.7 & $\ldots$ & $\ldots$ & 3240 & 27 \\
\hline $\mathrm{J} 1903+0327$ & Both & $-3.0(4)$ & $31(6)$ & $375(11)$ & 12 & 371 & 1.0 & 1456 & 99 \\
\hline J1909-3744 & Both & $-0.239(4)$ & $0.7(1)$ & $366(5)$ & 28 & 366 & 0.25 & 3306 & 9 \\
\hline $\mathrm{J} 1910+1256$ & Linear & $0.51(6)$ & $\cdots$ & $\cdots$ & 0.90 & 404 & 2.9 & 2574 & 443 \\
\hline $\mathrm{J} 1923+2515$ & None & $\cdots$ & $\cdots$ & $\cdots$ & $\cdots$ & $\cdots$ & $\cdots$ & 803 & $\cdots$ \\
\hline J1944+0907 & Linear & $1.3(2)$ & $\cdots$ & $\ldots$ & 44 & $\cdots$ & $\cdots$ & 1467 & 43 \\
\hline $\mathrm{J} 1949+3106$ & Periodic & $\ldots$ & $10(3)$ & $391(37)$ & 1.0 & $\cdots$ & $\cdots$ & 455 & 51 \\
\hline B1953+29 & Both & $-1.3(3)$ & $3(2)$ & $356(72)$ & 2.1 & $\cdots$ & $\cdots$ & 1967 & 136 \\
\hline J2010-1323 & Both & $0.38(2)$ & $2.2(4)$ & 372(9) & 14 & 372 & 0.56 & 1490 & 16 \\
\hline $\mathrm{J} 2017+0603$ & Both & $0.23(7)$ & $2.3(5)$ & $440(37)$ & 0.94 & $\cdots$ & $\cdots$ & 609 & 38 \\
\hline $\mathrm{J} 2043+1711$ & Both & $-0.12(4)$ & $1.0(4)$ & $390(38)$ & 3.7 & $\cdots$ & $\cdots$ & 834 & 189 \\
\hline $\mathrm{J} 2145-0750$ & Linear & $0.08(2)$ & $\cdots$ & $\ldots$ & 18 & $\ldots$ & $\ldots$ & 3318 & 568 \\
\hline $\mathrm{J} 2214+3000$ & Periodic & $\cdots$ & $4(1)$ & $319(25)$ & 0.83 & $\cdots$ & $\cdots$ & 755 & 17 \\
\hline $\mathrm{J} 2302+4442$ & Linear & $-0.6(2)$ & $\cdots$ & $\cdots$ & 1.5 & $\cdots$ & $\cdots$ & 613 & 202 \\
\hline $\mathrm{J} 2317+1439$ & Both & $-0.550(9)$ & $0.9(3)$ & $311(6)$ & 321 & $\cdots$ & $\cdots$ & 3243 & 5 \\
\hline PSR & Trend & $\begin{array}{c}d \mathrm{DM} / d t \\
\left(10^{-3} \mathrm{pc} \mathrm{cm}^{-3} \mathrm{yr}^{-1}\right)\end{array}$ & $\begin{array}{c}\text { Amplitude } \\
\left(10^{-4} \mathrm{pc} \mathrm{cm}^{-3}\right)\end{array}$ & $\begin{array}{l}\text { Period } \\
\text { (days) }\end{array}$ & $\chi_{r}^{2}$ & Start & End & $\begin{array}{l}\text { Length } \\
\text { (days) }\end{array}$ & $\begin{array}{c}\delta t \\
\text { (days) }\end{array}$ \\
\hline \multirow[t]{2}{*}{ J0613-0200 } & Both & $0.066(7)$ & $1.8(1)$ & $358(4)$ & 0.72 & 53448 & 54970 & 3137 & 18 \\
\hline & Both & $0.161(7)$ & $1.2(2)$ & $352(5)$ & 3.5 & 54970 & 56380 & $\cdots$ & 23 \\
\hline \multirow[t]{2}{*}{$\mathrm{J} 1600-3053$} & Linear & $-0.73(4)$ & $\cdots$ & $\cdots$ & 2.8 & 54400 & 55300 & 2184 & 40 \\
\hline & None & $\cdots$ & $\cdots$ & $\cdots$ & $\cdots$ & 55300 & 56585 & $\cdots$ & $\cdots$ \\
\hline \multirow[t]{2}{*}{$\mathrm{J} 1640+2224$} & Linear & $0.145(3)$ & $\cdots$ & $\cdots$ & 7.0 & 53344 & 55850 & 3254 & 78 \\
\hline & None & $\cdots$ & $\cdots$ & $\cdots$ & $\cdots$ & 55850 & 56599 & $\cdots$ & $\cdots$ \\
\hline \multirow[t]{3}{*}{$\mathrm{J} 1713+0747$} & Both & $-0.066(8)$ & $0.5(1)$ & $400(16)$ & 2.0 & 53393 & 54730 & 3199 & 38 \\
\hline & None & $\cdots$ & $\cdots$ & $\cdots$ & $\cdots$ & 54730 & 54900 & $\cdots$ & $\cdots$ \\
\hline & Both & $-0.015(5)$ & $0.52(9)$ & $369(7)$ & 5.5 & 54900 & 56592 & $\cdots$ & 26 \\
\hline \multirow[t]{2}{*}{$\mathrm{J} 1918-0642$} & Both & $-0.49(1)$ & $1.2(4)$ & $385(11)$ & 4.3 & 53292 & 56000 & 3293 & 24 \\
\hline & Both & $0.23(3)$ & $1.2(3)$ & $541(47)$ & 2.9 & 56000 & 56585 & $\cdots$ & 31 \\
\hline \multirow[t]{3}{*}{ B1937+21 } & Both & $-0.34(3)$ & $3.2(4)$ & $395(11)$ & 28 & 53267 & 54550 & 3327 & 5 \\
\hline & None & $\ldots$ & $\cdots$ & $\ldots$ & $\ldots$ & 54550 & 55970 & $\ldots$ & $\ldots$ \\
\hline & Both & $0.050(3)$ & $3.7(2)$ & $469(14)$ & 10 & 55970 & 56594 & $\ldots$ & 5 \\
\hline
\end{tabular}

Notes. Results of fitting periodic and linear trends to the DM variations, where $1 \sigma$ uncertainty in the last significant digit is expressed in parentheses. The upper section lists pulsars where a single fit was applied; columns are the detected trend, the slope, the amplitude of the periodic fit, the period of the fit, the reduced chi-squared after the fitting, the period found by the Lomb-Scargle periodogram, the false-alarm probability for that period, the length of the data span for that pulsar, and the average time it takes the DM time series to vary by $1 \sigma$. Values and corresponding errors were found by implementing a nonlinear least-squares Marquardt-Levenberg algorithm. Variations were determined to exhibit a trend if the post-fit $\chi_{r}^{2}$ was lower than the pre-fit $\chi_{r}^{2}$ value. The period found by the Lomb-Scargle periodogram analysis, $P_{\mathrm{LS}}$, was omitted if it corresponded to the length of the data set or the cadence of observations. The resolution of the Lomb-Scargle analysis (and hence error on $\left.P_{\mathrm{LS}}\right)$ is equal to the cadence of the observations for that particular pulsar. Those MSPs that are identified as having a linear trend had that trend subtracted off prior to applying the periodogram analysis. The lower section contains pulsars where piecewise trends were applied. The two columns that differ from above give the final start and end dates used in the fit. The Lomb-Scargle periodogram found a period for PSR J0613-0200 of 371 days with a false-alarm probability of 5.4\%.

estimating the number of degrees of freedom for a nonlinear model (Andrae et al. 2010). The $\chi_{r}^{2}$ is only used as a metric to compare the fits of models we know to be incomplete; as stated earlier, the ISM is more complicated than a purely linear trend plus annual component. The fitting routine incorporates a nonlinear least-squares fit that is locally linearized around the 
minimum $\chi_{r}^{2}$. Later on, we describe $\chi_{r}^{2}$ surfaces in the full parameter space and analyze the degree of covariance between fit parameters, finding it agrees with this fitting routine.

The periodic term in the function was fit using an initial guess of 365 days. Due to a change in sign of $d \mathrm{DM} / d t$ or the appearance or disappearance of a trend partway through the data span, PSRs J0613-0200, J1600-3053, J1640+2224, J1918-0642, and B1937+21 are not well characterized by a single fit. These MSPs were fit using piecewise functions, using the $\chi_{r}^{2}$ of the fit to identify the applicable MJD range for each fit. The results of this partial fitting can be seen in Table 2. The fits can be seen in Figures 1 through 5. The $\chi_{r}^{2}$ values listed in Table 2 are for the individual fit regions; these differ from the values shown in Figures 1 through 5 because those values incorporate both fits as well as any regions excluded from the fit.

We applied a Lomb-Scargle periodogram analysis to corroborate the best-fit periods, as an annual period was suggested during the trend-fitting routine. The periodogram is also useful in possibly identifying nonannual periodicities (also seen in Table 2). This analysis is able to detect periodicities in unevenly sampled data (Scargle 1982) for which a false-alarm probability (FAP) may be calculated. The FAP is the likelihood that these periods would occur as a result of random white noise. We ignored periods found by the periodogram that coincided with either the length of the data set or the observing cadence. The resolution of the analysis is equal to the cadence of the observations. Any linear trend in the DM variations will mask the periodic effect and therefore was removed from those identified to have linear effects before applying the periodogram analysis.

\subsection{DM Variation Timescale}

The DM value can vary on timescales of years, days, or even hours. Therefore it is important to know on what timescale this $\mathrm{DM}$ is accurate. The time $\delta t$ for $\mathrm{DM}$ to change by $\sigma_{\mathrm{DM}}$, the rms $\mathrm{DM}$ in the DM time series (prior to fitting any DM trends), gives us a rough estimate for how long a single DM estimation is valid. For a linear trend,

$$
\frac{\sigma_{\mathrm{DM}}}{\delta t}=\frac{d \mathrm{DM}}{d t}=m \longrightarrow \delta t=\frac{\sigma_{\mathrm{DM}}}{m},
$$

where $m$ is the slope of the linear trend, seen also in Equation (4). The time associated with a periodic trend

$$
\frac{\sigma_{\mathrm{DM}}}{\delta t}=\frac{d \mathrm{DM}}{d t} \approx A \omega \longrightarrow \delta t=\frac{\sigma_{\mathrm{DM}}}{A \omega},
$$

where $A$ and $\omega$ are the amplitude and angular frequency of the periodic trend, respectively. The variation time for a DM time series showing both trends combines the $d \mathrm{DM} / d t$ components from both the periodic and linear components:

$$
\frac{\sigma_{\mathrm{DM}}}{\delta t} \approx m+A \omega \longrightarrow \delta t \approx \frac{\sigma_{\mathrm{DM}}}{m+A \omega}
$$

The $\delta t$ values for the MSPs showing trends are seen in Tables 2. This $\delta t$ can inform on what timescale our DM measurement is constant and the importance of observing at epochs with spacing smaller than this timescale.

\subsection{Solar-angle Correlation}

Pulsars that lie close to the ecliptic (within approximately $10^{\circ}$ ) will have their LOS pass near the Sun during Earth's orbit. This proximity can cause a sinusoidal trend in DM variations due to the variation in $n_{e}$ along the LOS from the solar wind.

We examine the pulsar positions with respect to the ecliptic to determine for which MSPs this effect could be significant. As can be seen in Figure 6, PSRs J0023+0923, J0030+0451, J1614-2230, and J2010-1323 reside close (within approximately $6.3,1.5,6.8$, and 6.5 , respectively, on closest approach in the data set) to the ecliptic. The DM as a function of solar elongation angle can be seen in Figure 7. PSRs J0023+0923 and J2010-1323 show a slight peak in DM at the smallest pulsarSun angles. PSRs J0030+0451 and J1614-2230 show significant peaks at the minimal solar elongation angle. It should be noted that the two highest DM points for $\mathrm{J} 0030+0451$ were omitted from Arzoumanian et al. (2015) as outliers but were included for this portion of the analysis.

\subsection{Pulsar Trajectories}

We have plotted the pulsar trajectories through the ISM as seen from Earth, color coded to signify the DM value at each epoch (Figures 8 through 11). For this, we assumed that all of the free electrons along the LOS are sitting in a stationary phase screen located halfway between the Earth and the pulsar. The trajectories are the projected motions of the pulsar as seen on this phase screen. Using proper motion and distance estimates with errors from the NE2001 model (Cordes \& Lazio 2002), the transverse velocity can be calculated and used to track the pulsar's trajectory in the sky. Proper motions were taken from the data release (seen in Table 1). These trajectory maps can be useful in isolating features in the ISM as well as visualizing trends in the DM time series.

\section{Structure Functions}

Turbulence in the ISM is typically described as having a Kolmogorov power spectrum, meaning we expect to find larger variations over longer timescales. The power spectrum used to derive the SF has the form

$$
P(q) \propto q^{-\beta}, q_{\text {outer }} \leqslant q \leqslant q_{\text {inner }},
$$

where $q$ is the reciprocal of the size scale, and $\beta$ is the power spectrum exponent. A Kolmogorov medium corresponds to a $\beta$ value of $11 / 3$, while the highest value expected for turbulence in the ISM (for an inner scale shorter than $10^{9} \mathrm{~m}$ ) is $\beta=4$ (Rickett 1990). The outer scale is described as the size at which the ISM ceases to be homogeneous, and the inner scale is the point at which dissipation occurs in the material along the LOS.

The DM SF is an effective analytical tool for characterizing interstellar turbulence over various time and size scales (Cordes et al. 1990; Rickett 1990; You et al. 2007; Keith et al. 2013; Fonseca et al. 2014; Lam et al. 2016). We compute SFs by binning the change in time across all epochs into equally logspaced bins after calculating

$$
D_{\mathrm{DM}}(\tau)=\left\langle[\mathrm{DM}(t+\tau)-\operatorname{DM}(t)]^{2}\right\rangle,
$$

where $\tau$ is the time lag in days. The diffractive timescale $\Delta \tau_{D}$, the scale during which the diffraction intensity varies as a result of irregularities in the ionized plasma along the LOS, is used to 

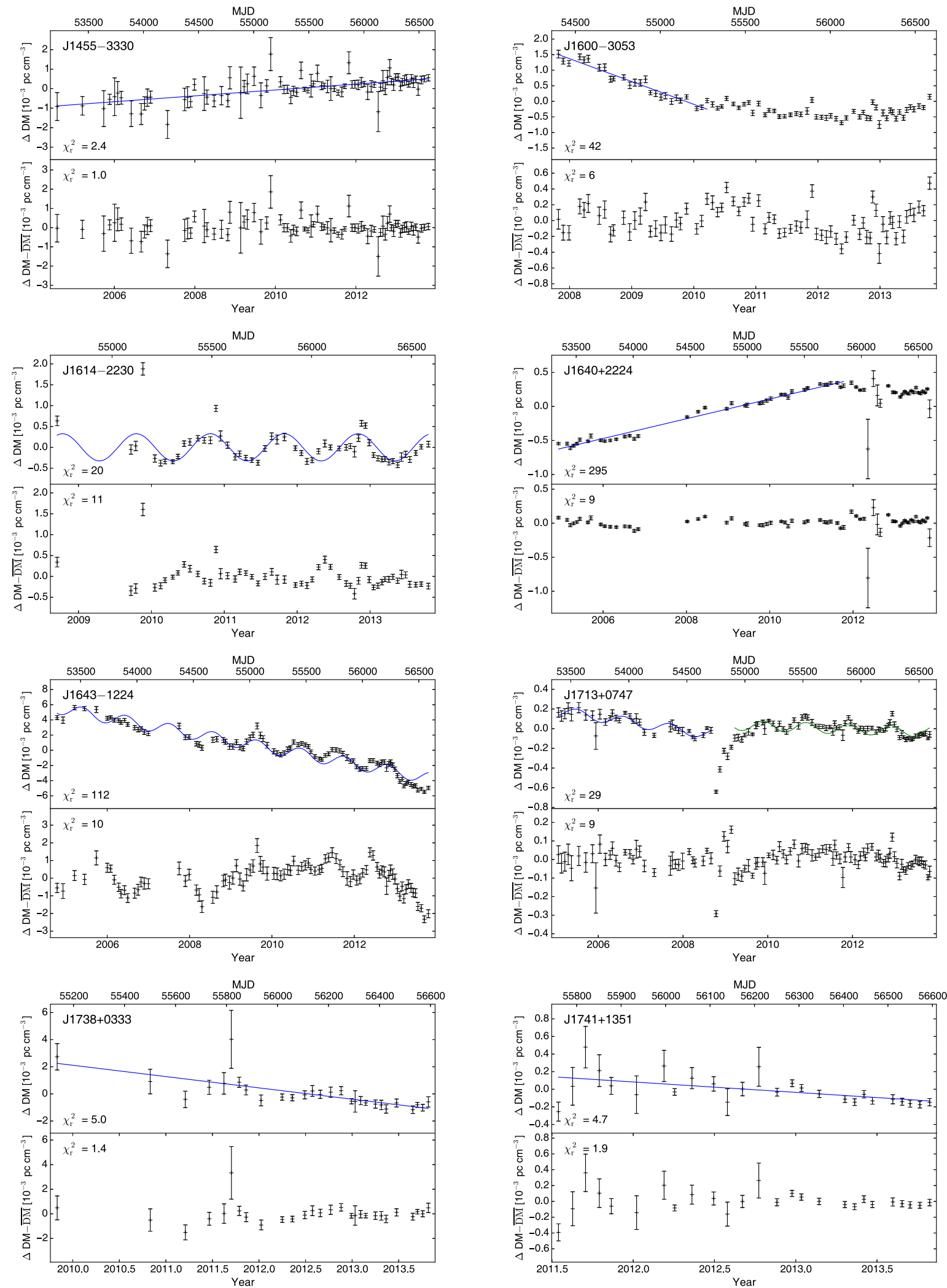

Figure 2. The top panel shows the DM time series with the best-fit function (if applicable) in blue. The zero point for the DM variations corresponds to the fiducial DM for the data span. The error bars are $\pm 1 \sigma$ errors returned by TEMPO. The bottom panel shows the DM residuals after the trend has been removed from the time series; an empty panel means no trend was found. The $\chi_{r}^{2}$ values before and after these fits for each pulsar appear in the top and bottom panels, respectively, as well as in Tables 1 and 2. PSRs J1600-3053 and J1640+2224 were not found to have significant trends in the later parts of the DM time series. Partial DM $(t)$ ranges were published in Levin et al. (2016) for PSRs J1614-2230, J1713+0747, J1738+0333, and J1741+1351. 

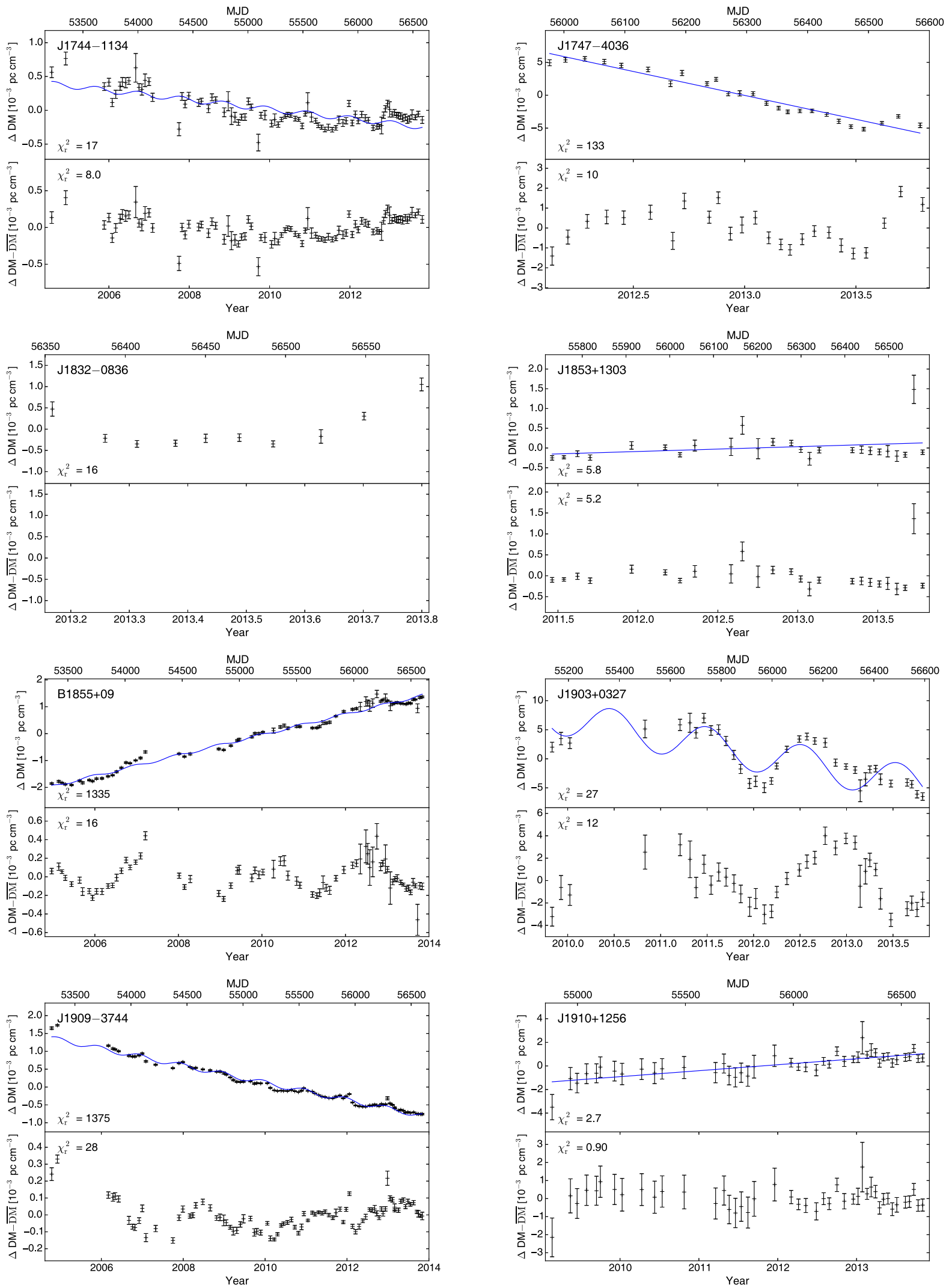

Figure 3. The top panel shows the DM time series with the best-fit function (if applicable) in blue. The zero point for the DM variations corresponds to the fiducial DM for the data span. The error bars are $\pm 1 \sigma$ errors returned by TEMPO. The bottom panel shows the DM residuals after the trend has been removed from the time series; an empty panel means no trend was found. The $\chi_{r}^{2}$ values before and after these fits for each pulsar appear in the top and bottom panels, respectively, as well as in Tables 1 and 2. PSR J1832-0836 has too short a data span for a trend to be determined. Partial DM( $t$ ) ranges were published in Levin et al. (2016) for PSRs $\mathrm{J} 1744-1134, \mathrm{~B} 1855+09$, J1909-3744, and $\mathrm{J} 1910+1256$. 

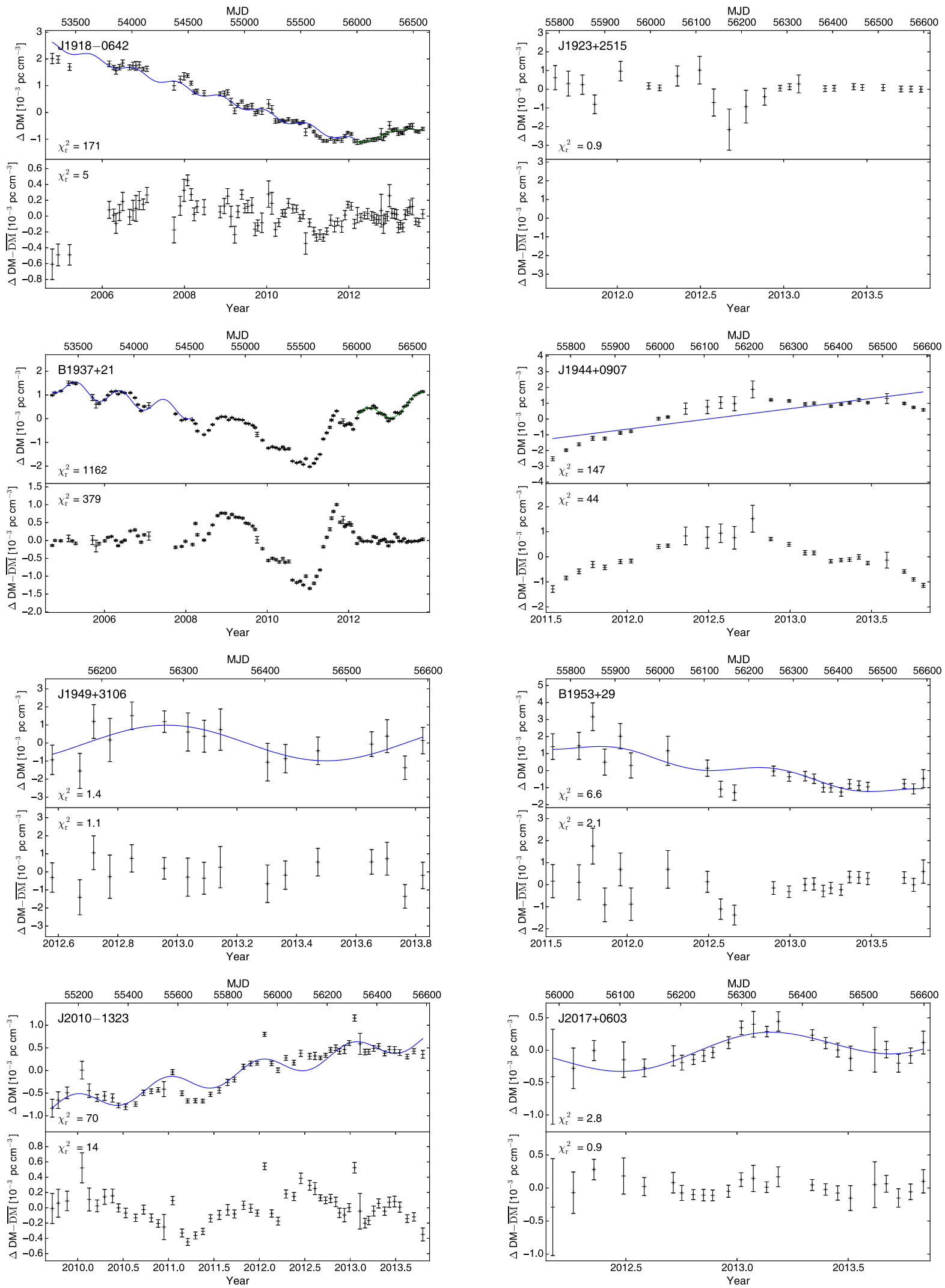

Figure 4. The top panel shows the DM time series with the best-fit function (if applicable) in blue. The zero point for the DM variations corresponds to the fiducial DM for the data span. The error bars are $\pm 1 \sigma$ errors returned by TEMPO. The bottom panel shows the DM residuals after the trend has been removed from the time series; an empty panel means no trend was found. The $\chi_{r}^{2}$ values before and after these fits for each pulsar appear in the top and bottom panels, respectively, as well as in Tables 1 and 2. PSR B1937+21 could not be fit with a periodic trend throughout the data set. Partial DM( $t)$ ranges were published in Levin et al. (2016) for PSRs J1918-0642, B1937+21, J1944+0907, and J2010-1323. 

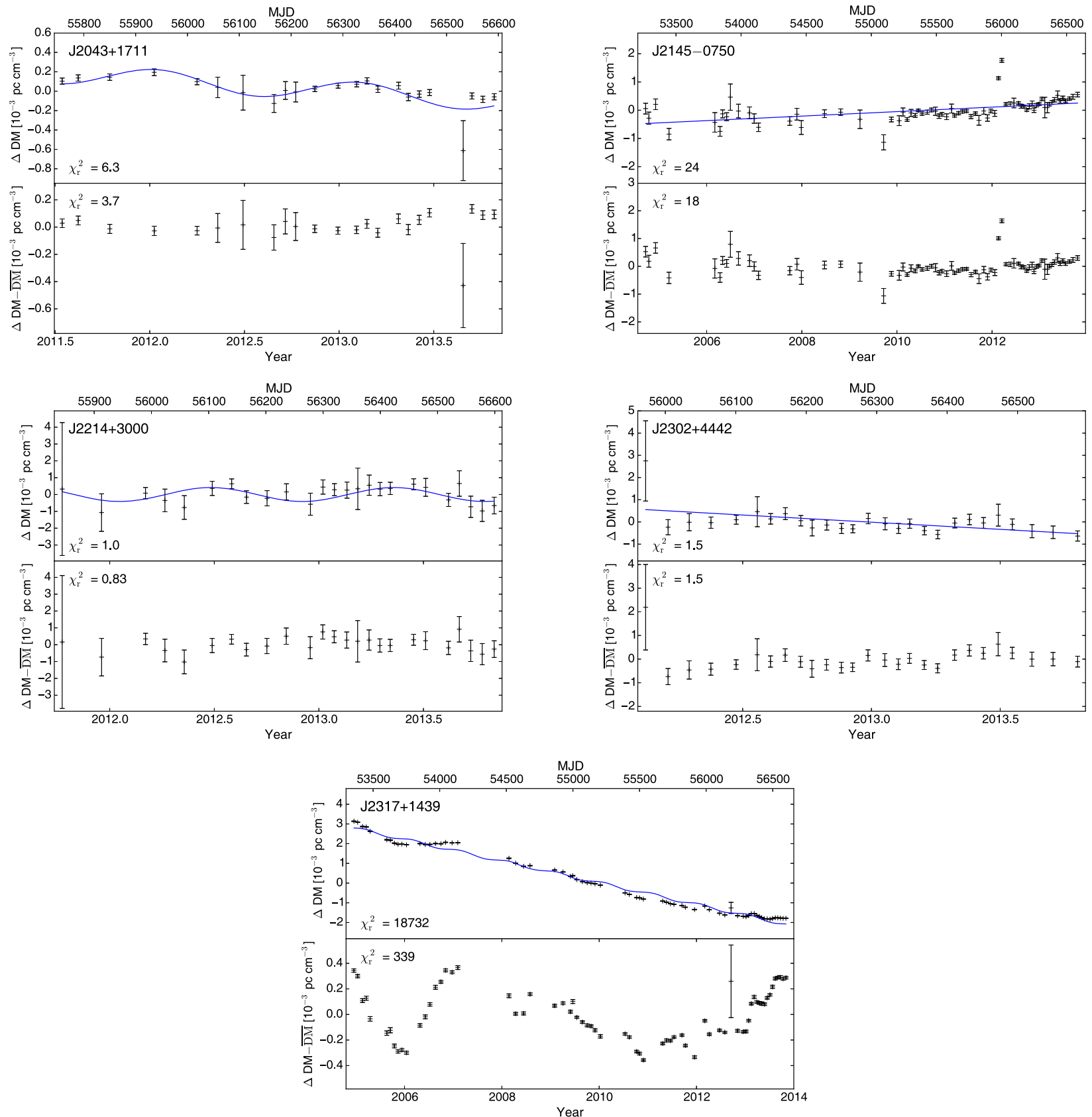

Figure 5. The top panel shows the DM time series with the best-fit function (if applicable) in blue. The zero point for the DM variations corresponds to the fiducial DM for the data span. The error bars are $\pm 1 \sigma$ errors returned by TEMPO. The bottom panel shows the DM residuals after the trend has been removed from the time

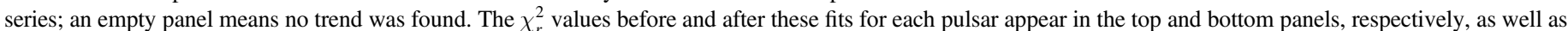
in Tables 1 and 2. Partial DM( $t$ ) ranges were published in Levin et al. (2016) for PSR 2302+4442.

anchor the SF:

$$
D_{\mathrm{DM}}(\tau)=\left(A_{\nu} \nu\right)^{2}\left(\tau / \Delta \tau_{\mathrm{D}}\right)^{\beta-2},
$$

where $A_{\nu}=3.84 \times 10^{-5} \mathrm{MHz}^{-2} \mathrm{~s}^{-1} \mathrm{pc} \mathrm{cm}^{-3}$ and $\nu$ is the observing frequency; the somewhat unintuitive units on $A_{\nu}$ arise because of the relationship to the phase SF, which is used to constrain the DM SF (Rickett 1990; Fonseca et al. 2014). Epoch-to-epoch variations of $\Delta \tau_{\mathrm{D}}$ are expected to be of order $10 \%$ or more (Lam et al. 2016). The SF is poorly estimated at large time lags, so some functions may appear Kolmogorov at shorter timescales but fall below at longer time lags; this is an indication of an underlying Kolmogorov spectrum (You et al. 2007). This is also why SF values at high time lags may have large errors on them. Quadratic SFs occur when the time lag is smaller than the timescale necessary to adequately probe the structure in a region, if any (Lam et al. 2016).

Several models were applied to the SF in order to constrain a range for $\beta$. Fitting only for Equation (10) is problematic because there are other contributions to the SF, such as trends and noise, among other possibilities. However, overfitting the $\mathrm{SF}$ will cause the fit to fail for a number of reasons, discussed in this section. The models are of the form

$$
D_{\text {DM }}(\tau)=D_{\text {sto }}(\tau)+D_{\text {lin }}(\tau)+D_{\text {per }}(\tau)+D_{\text {noise }}(\tau),
$$

where the first term is the stochastic (e.g., from the electron density wavenumber spectrum) component, the second term is 

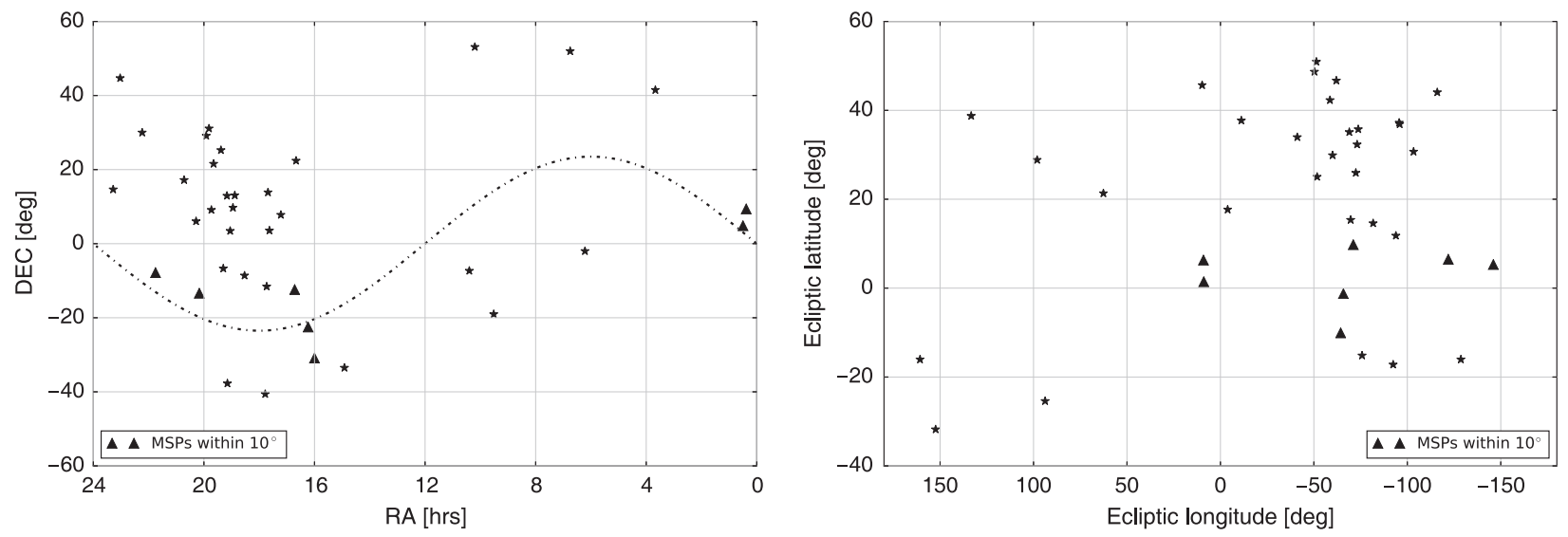

Figure 6. MSP positions with respect to the ecliptic, shown in R.A. and decl. (left) as well as ecliptic coordinates (right). In the left plot, the ecliptic is depicted by the dashed line. Sources that lie within $\sim 10^{\circ}$ of the ecliptic are signified by triangles. A number of pulsars pass near enough to the Sun for the line of sight to pass through the densest parts of the solar wind, resulting in a variation in the number of free electrons over an annual cycle.

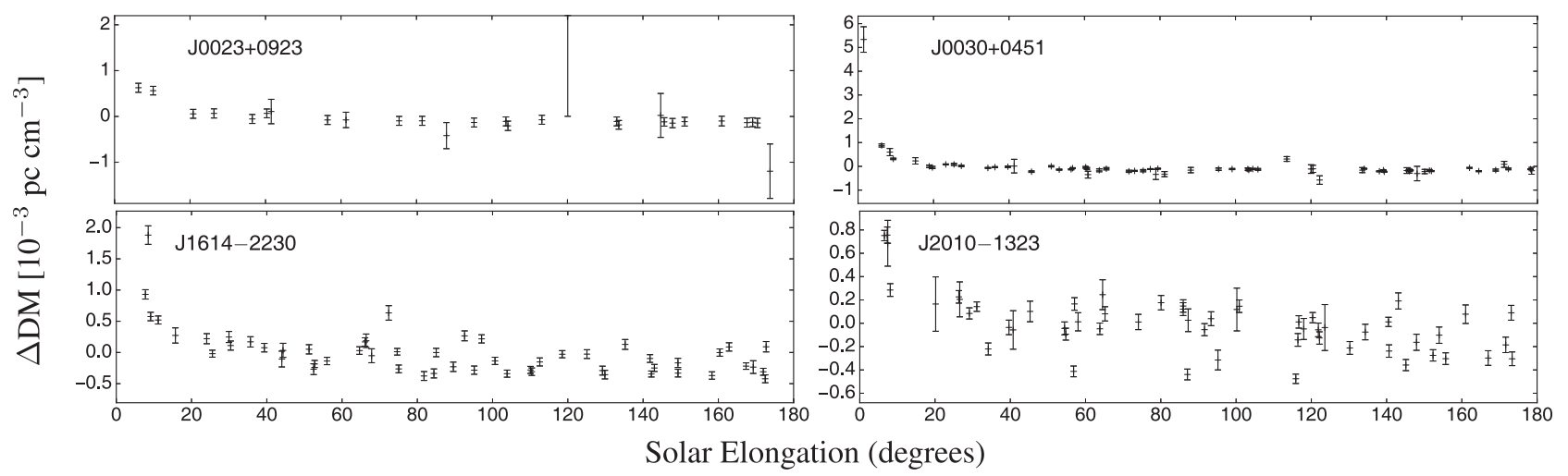

Figure 7. DM variations with respect to the solar elongation. The linear trend identified for PSR J2010-1323 has been subtracted in order to better identify any correlation in the DM as a function of solar elongation. Note that the two highest DM points seen for PSR J0030+0451 were omitted from the nine-year data set as outliers and are not plotted in Figure 1 (discussed in Section 3.3).

the linear component, the third term is the periodic component, and the last term is the noise (e.g., measurement error) component.

One method is to fit the stochastic and noise components while fixing the linear trend and periodic components to the trend values found in the time series analysis. However, we were only able to successfully fit the SF for one pulsar (PSR J1643-1224) using this model. In all other cases, the SF of the two trends is higher than the calculated SF; fitting for a stochastic component on top of that would only increase the chi-squared, and the fit fails. This failure is evidence that there is contamination between the stochastic and trend components. In many cases, a linear trend fit over the time series will absorb part of the stochastic component. Therefore, the "true" linear trend may be different than the one we fit for in the time series, which will bias the component in the SF high. Therefore, we are unable to obtain a proper fit using this model.

To bypass this contamination between the trend and stochastic aspects, we tried fitting for all parameters (stochastic, noise, linear, and periodic) without using any prior information in the hopes of allowing a fit and comparing values with those previously found. Due to the covariant nature of the parameters, we found values for the stochastic, periodic amplitude, and noise components, but the amplitude of the periodic trend was not much larger than its uncertainties, while the linear trend component was found to be zero. The period was then fixed to one year in order to eliminate some of the covariance; fixing the period again gave significant values for the stochastic, noise, and periodic amplitude components and no significance for an additional linear component. This is further evidence of the high covariance between the stochastic and linear trend components. The periodic amplitudes found here agree very well with those found previously by the trend analysis. Simultaneously fitting both the stochastic and linear components does not appear to yield significant values for both.

The simplest model is then to only fit for stochastic and noise components while not fitting for a linear trend component. This model can be applied to all pulsars. Because the periodic component was not found to be highly covariant with the stochastic component, the periodic amplitudes were fitted as well and compared to the values from the trend analysis. In order to constrain a linear trend, we would need some prior information on the shape of the stochastic component in the time series relative to the true linear component that we do not have at this time.

Diffractive timescales, listed in Table 3, were calculated by creating a two-dimensional dynamic spectrum of each $1500 \mathrm{MHz}$ observation in the data set and computing the 2D autocorrelation function of each spectrum, which in turn is summed over time and frequency separately. A Gaussian function is fitted to the $1 \mathrm{D}$ frequency-summed autocorrelation 

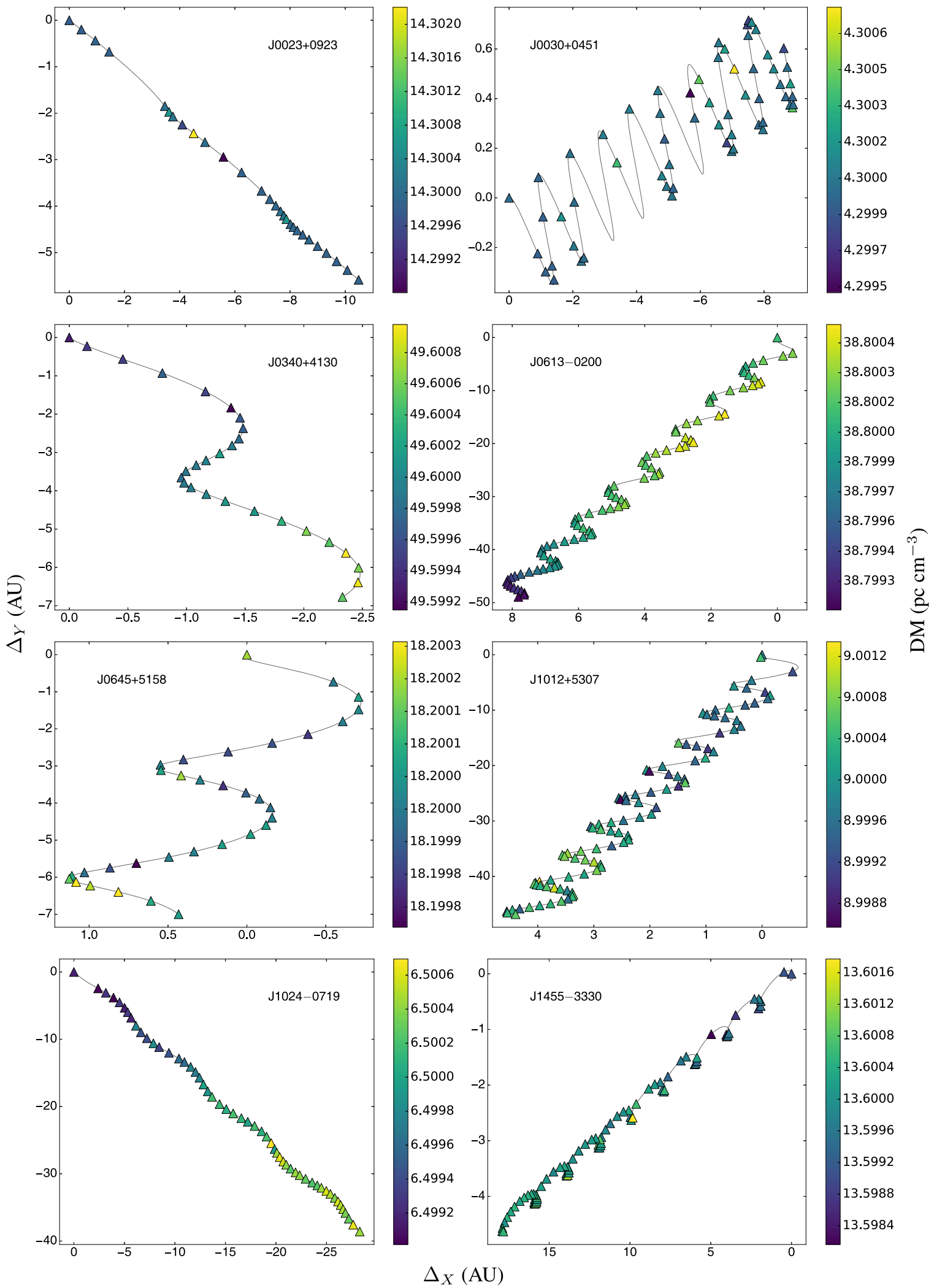

Figure 8. MSP trajectories are plotted with color mapping the DM at each epoch. The trajectories are calculated assuming that all the free electrons along the LOS are sitting in a phase screen halfway between the Earth and the MSP; the trajectory is then the projected motion of the pulsar on the phase screen. The axes depict the space traversed at the phase screen in au in the R.A. and decl. directions. The pulsar's motion starts at $(0,0)$. Pulsars closer to the ecliptic will show a tighter sinusoid than those farther away. The trajectory plot can be used to show limited localized structure. For example, PSR J0613-0200 shows higher DM regions on one side of its trajectory over time, suggesting the presence of a DM gradient that is transverse compared to the pulsar's motion. PSR J1024-0719 shows a monotonically increasing DM, which could be due to a DM gradient oriented in the same direction as the pulsar's motion. 

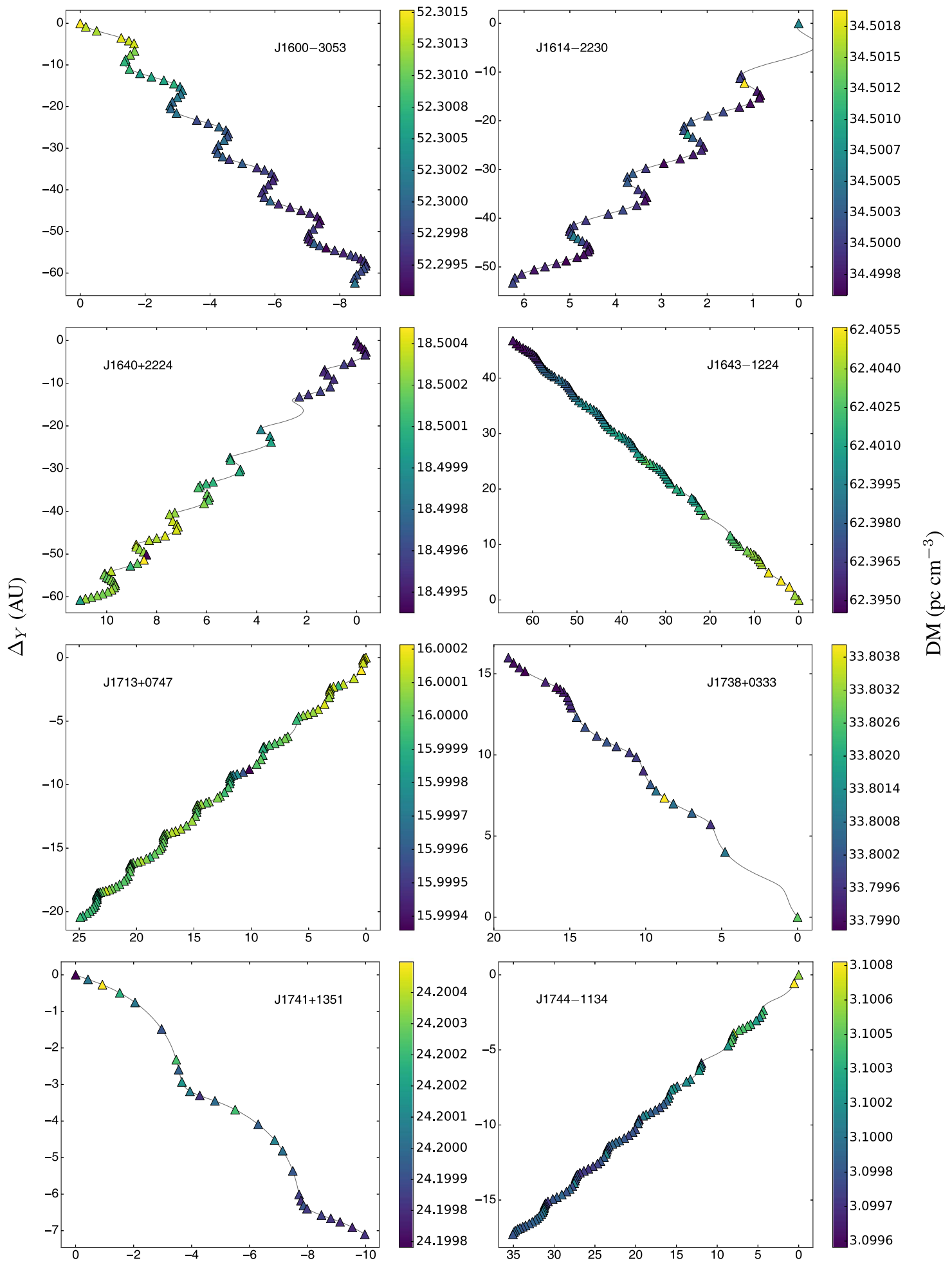

$\Delta_{X}(\mathrm{AU})$

Figure 9. MSP trajectories are plotted with color mapping the DM at each epoch. The trajectories are calculated assuming that all the free electrons along the LOS are sitting in a phase screen halfway between the Earth and the MSP; the trajectory is then the projected motion of the pulsar on the phase screen. The axes depict the space traversed at the phase screen in au in the R.A. and decl. directions. The pulsar's motion starts at $(0,0)$. Pulsars closer to the ecliptic will show a tighter sinusoid than those farther away. The trajectory plot can be used to show limited localized structure. PSRs J1600-3053, J1640+2224, and J1643-1224 show a monotonically increasing or decreasing DM, which could be due to a DM gradient along the direction of motion. PSR J1614-2230 has high DM regions in the same part of its trajectory every year, suggesting a localized overdensity along the line of sight to the pulsar.

function, and the scintillation timescale is defined as the halfwidth at $e^{-1}$. This is following the same procedure as described in Levin et al. (2016).
Most observations in the nine-year data set are around 30 minutes long, and for many pulsars, the scintillation timescale is much longer than this integration time. Therefore, it was only 

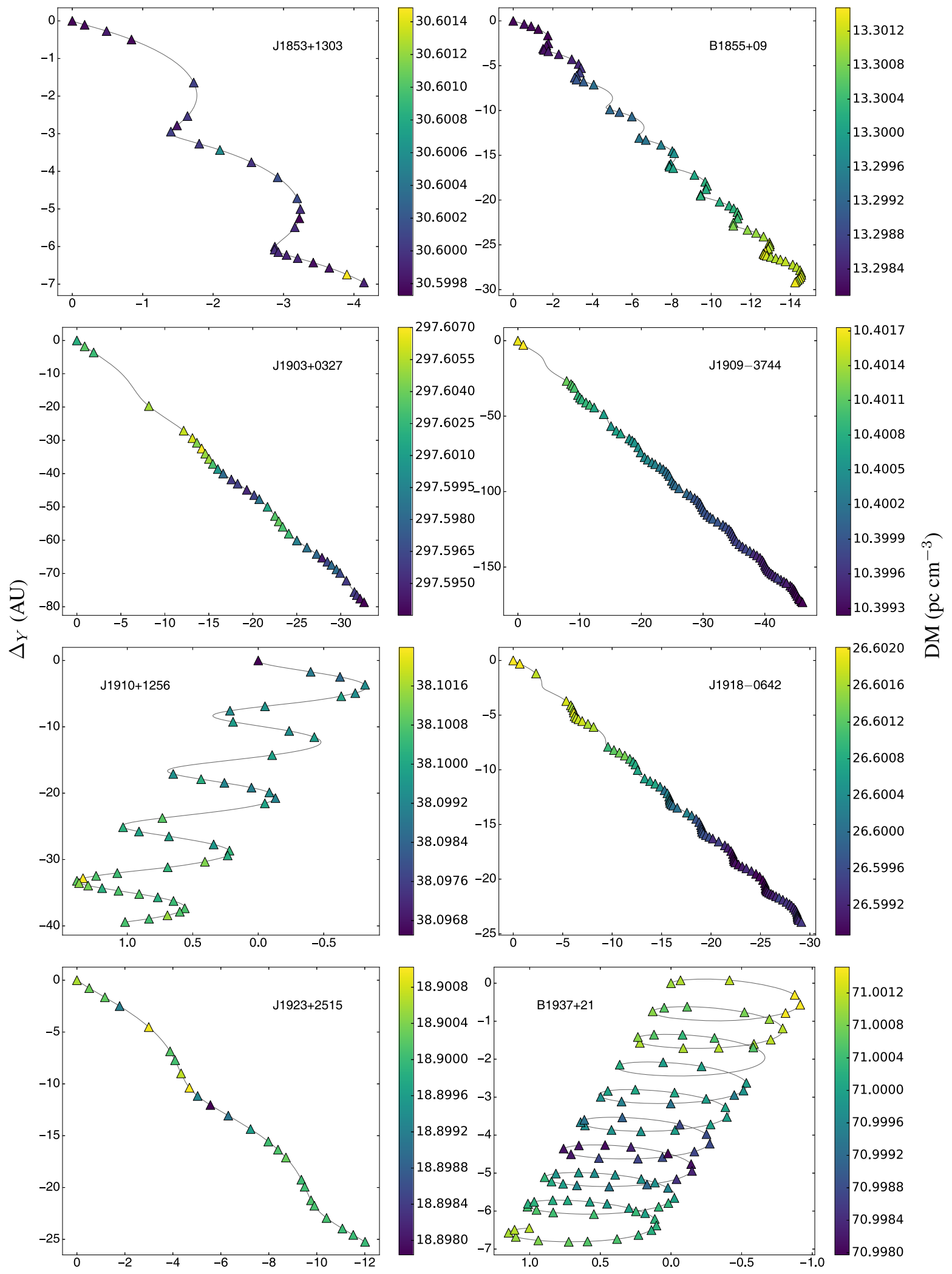

$\Delta_{X}(\mathrm{AU})$

Figure 10. MSP trajectories are plotted with color mapping the DM at each epoch. The trajectories are calculated assuming that all the free electrons along the LOS are sitting in a phase screen halfway between the Earth and the MSP; the trajectory is then the projected motion of the pulsar on the phase screen. The axes depict the space traversed at the phase screen in au in the R.A. and decl. directions. The pulsar's motion starts at $(0,0)$. Pulsars closer to the ecliptic will show a tighter sinusoid than those farther away. The trajectory plot can be used to show limited localized structure. PSRs B1855+09, J1909-3744, and J1918-0642 show a monotonically increasing or decreasing DM, which could be due to a DM gradient along the direction of motion. PSR J1903+0327 has high DM regions in the same part of its trajectory every year, suggesting a localized overdensity along the line of sight to the pulsar. PSR B 1937+21 shows a distinct low DM region that is also evident in the DM time series, indicative of a localized bubble in the ISM. 
Table 3

Diffractive Timescales for 18 MSPs

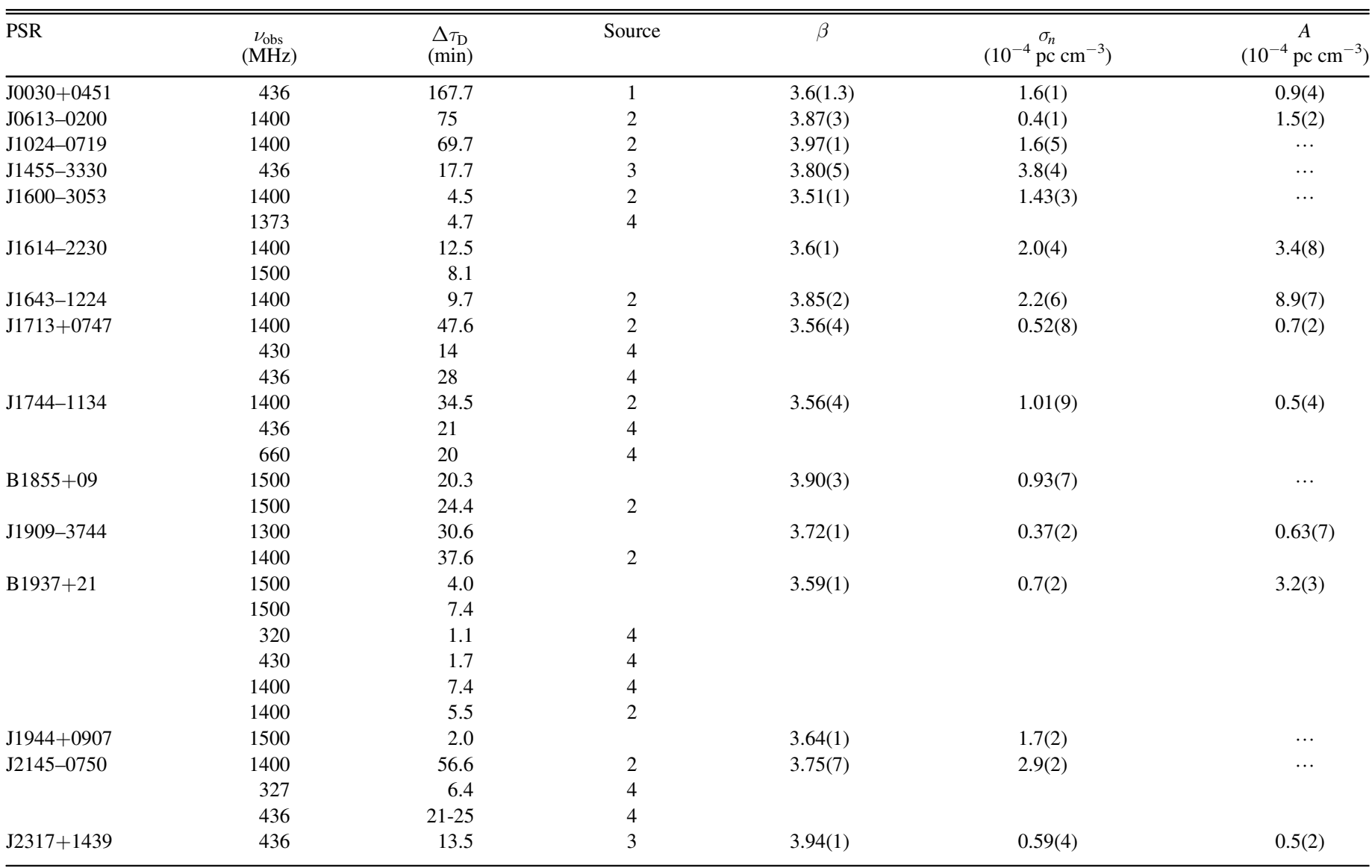

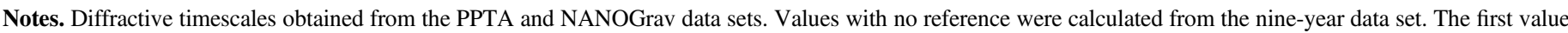

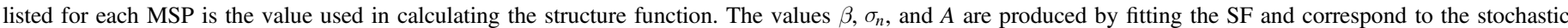

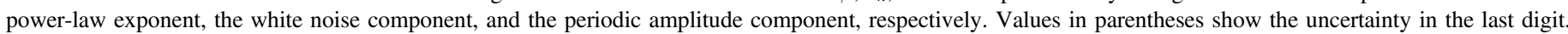
References. (1) Nicastro et al. (2001), (2) Keith et al. (2013), (3) Johnston et al. (1998), (4) You et al. (2007).

possible to measure diffractive timescales in this way for a few of the pulsars in the sample.

Lag bins are equally spaced in log space. Errors on the SF were calculated by combining the propagated errors from the DM time series values and the errors due to uncertainty in the specific realizations in a stochastic process. To constrain those errors, we used simulations of different spectral slopes and different time spans of data, and the standard deviation of the SF was calculated for $10^{4}$ realizations at every time lag $\tau$. This was saved as a $3 \mathrm{D}$ grid of values (time $\operatorname{span}, \beta, \tau$ ), and then a function was used to interpolate over that grid to give the realization error of the SF value at each $\tau$. We held $\beta$ constant to the value for a Kolmogorov medium because varying the slope had a negligible effect on the derived errors.

\section{Results}

\subsection{Linear Trends and Annual Periodicities}

Of the 37 MSPs in the data release, 33 show the presence of DM trends, of which six MSPs show piecewise trends over different time spans. With the least-squares fitting procedure, we find periods roughly consistent with an annual periodicity of \pm 54 days for 18 pulsars; seven of these periods were also detected by the periodogram. The periodogram found significant periods that were not consistent with annual for two pulsars (PSRs J0645+5158 and J1747-4036). A period of 199 days was found for J0645+5158; this roughly semiannual period could be indicative of several effects in the DM time series (ionosphere, refractive effects from a clump of material along the LOS, or a solar wind event, among other possibilities; Lam et al. 2016). More investigation (and probably more data) would be needed to more definitively identify the source. The power spectrum, seen in Figure 14, shows a significant peak at the found period of 199 days and a secondary much less significant annual period, where the uncertainty in both of these periods is equivalent to the cadence of observations. A period of 459 days was found for J1747-4036, but there are less than two years of data for this pulsar, so this periodicity may not be robust. Keith et al. (2013) predicted that annual modulations would be seen based on the spectral analyses for PSRs J1024-0719 and J1909-3744 and that they are dominated by the steep linear trend. The authors suggest that a more significant detection could occur by combining data sets. Our trend analysis did not find an annual trend in PSR J1024-0719. We did, however, calculate a period of $366 \pm 5$ days for PSR J1909-3744. A linear trend was found in 28 pulsars, 14 of which also exhibit annual trends. Five pulsars exhibit annual trends without a linear trend.

One possible cause of linear trends in $\operatorname{DM}(t)$ is an increasing or decreasing distance between the Earth and the pulsar. For four of 
Table 4

Positions and Corrected Velocities for Three MSPs

\begin{tabular}{lrcrr}
\hline \hline PSR & $l$ & $b$ & $\begin{array}{r}v_{E} \\
\left(\mathrm{~km} \mathrm{~s}^{-1}\right)\end{array}$ & \multicolumn{1}{c}{$\begin{array}{r}v_{p} \\
\left(\mathrm{~km} \mathrm{~s}^{-1}\right)\end{array}$} \\
\hline $\mathrm{J} 1012+5307$ & 160.35 & 50.86 & $-6.1(3)$ & $38(8)$ \\
$\mathrm{J} 1024-0719$ & $251^{\circ} .70$ & 40.52 & $-15.8(8)$ & $205(30)$ \\
$\mathrm{J} 1738+0333$ & $27^{\circ} .72$ & 17.74 & $16.8(8)$ & $-25(16)$ \\
$\mathrm{J} 1903+0327$ & $37^{\circ} .34$ & -1.01 & $16.0(8)$ & $58(3)$ \\
\hline
\end{tabular}

Note. Columns are Galactic longitude and latitude of the pulsar, the LOS component of the solar velocity, and the corrected velocity of the pulsar with respect to its local medium. Galactic coordinates were taken from the ATNF Pulsar Catalogue (Manchester et al. 2005, http://www.atnf.csiro.au/people/pulsar/ psrcat/).

the pulsars in the nine-year data set, LOS velocities have been measured with high precision. This allows us to solve for the free electron density around the pulsar. Matthews et al. (2016) calculated the velocity along the LOS for PSRs J1012+5307 and $\mathrm{J} 1903+0327\left(44.0 \pm 8.0 \mathrm{~km} \mathrm{~s}^{-1}\right.$ and $42.1 \pm 2.50 \mathrm{~km} \mathrm{~s}^{-1}$, respectively), Kaplan et al. (2016) found a LOS velocity of $221 \pm 30 \mathrm{~km} \mathrm{~s}^{-1}$ for J1024-0719, and Antoniadis (2013) found a velocity of $-42 \pm 16 \mathrm{~km} \mathrm{~s}^{-1}$ for $\mathrm{J} 1738+0333$. We correct these for the Sun's motion by calculating the Sun's velocity with respect to the local medium along each of the three lines of sight, given a velocity of $18.0 \pm 0.9 \mathrm{~km} \mathrm{~s}^{-1}$ in the direction $l=47^{\circ} .9 \pm 3.0$ and $b=23.8 \pm 2.0$ (Frisch et al. 2011). The corrected pulsar velocities are given in Table 4. Following Lam et al. (2016), we calculate the corresponding free electron density for each linear trend in DM as

$$
\frac{d \mathrm{DM}}{d t}=n_{e}\left(\boldsymbol{x}_{p}\right) v_{p}-n_{e}\left(\boldsymbol{x}_{E}\right) v_{E}
$$

where $n_{e}\left(\boldsymbol{x}_{p}\right)$ and $n_{e}\left(\boldsymbol{x}_{E}\right)$ are the free electron densities at the Earth and the pulsar, and $v_{p}$ and $v_{E}$ are the LOS velocities of the Earth and the pulsar through their local media. We calculate $n_{e}=2.8 \pm 0.8 \mathrm{~cm}^{-3}$ and $n_{e}=1.9 \pm 0.3 \mathrm{~cm}^{-3}$ for PSRs J1012 +5307 and J1024-0719, respectively. These are higher than the typical value for our Galaxy $\left(n_{e} \approx 0.03 \mathrm{~cm}^{-3}\right)$, but local environments can vary drastically, so the linear trends present could be explained by the LOS velocities and could indicate increased ionization as the pulsar is moving away from us. For comparison, Lam et al. (2016) find $n_{e}=7.6 \pm 2.9 \mathrm{~cm}^{-3}$ around PSR J1909-3744 following the same method. For PSR J1738 +0333 , we find $n_{e}=31 \pm 21 \mathrm{~cm}^{-3}$, which is much higher than a typical value but with high uncertainties given the uncertainties on the pulsar velocity and $d \mathrm{DM} / d t$. For PSR J1903+0327, we calculate a negative $d \mathrm{DM} / d t$, which is not as expected because the pulsar is moving away from us. Using a typical $n_{e}$, we would expect a decreasing linear trend of order $d \mathrm{DM} / d t \approx-7 \times$ $10^{-7} \mathrm{pc} \mathrm{cm}^{-3} \mathrm{yr}^{-1}$. Therefore, the DM variations must be dominated by another effect (such as refractive lensing, which may also explain the quasi-periodicities seen for this pulsar; Lam et al. 2016).

Reardon et al. (2016) modeled the DM variations for 20 PPTA-observed MSPs, nine of which are also included in the NANOGrav nine-year data release (PSRs J0613-0200, J1024 -0719, J1600-3053, J1643-1224, J1744-1134, B1855+09, J1909-3744, B1937+21, and J2145-0750). The trend assignments agree between the NANOGrav and PPTA data for PSRs 1024-0719, J1600-3053, J1643-1224, J1713+0747,
Table 5

Significance of DM Peaks for MSPs within $10^{\circ}$ of the Ecliptic

\begin{tabular}{lccc}
\hline \hline PSR & $\begin{array}{c}\sigma \\
\left(10^{-3} \mathrm{pc} \mathrm{cm}^{-3}\right)\end{array}$ & $\mathrm{DM}_{\text {peak }} / \sigma$ & $\theta$ \\
\hline J0023+0923 & 0.53 & 1.2 & 6.3 \\
J0030+0451 & 0.12 & 44.4 & 1.5 \\
J1614-2230 & 0.22 & 4.3 & 6.8 \\
J2010-1323 & 0.18 & 4.2 & 6.5 \\
\hline
\end{tabular}

Note. Columns are the rms $\sigma$ of DM measurements with a solar elongation greater than $30^{\circ}$, the ratio of the highest DM value in the data set over the rms, and the minimal angle $\theta$ between the Sun and the pulsar in the nine-year data. The rms was calculated using only DM measurements with $\theta>30^{\circ}$ to avoid contamination from the peak DM value.

B1937+21, and J2145-0750. The PPTA data did not show a linear trend for PSR J0613-0200 or periodic trends for PSRs B1855+09 and J1909-3744. Discrepancies could arise due to the difference in data spans, trends that continue or vary after the end of the data set, variations in methods of DM fitting, as well as differences in the trend-fitting algorithm; we fit for the period, whereas it is fixed at one year by Reardon et al. (2016). There is also the possibility that the DMs measured for the observed epochs do not agree between the sites due to the difference in observing frequencies used and spatial location; the two data sets may essentially be sampling a different ISM due to multipath scattering (Cordes et al. 2016).

Lentati et al. (2016) apply a Fourier-based method of DM estimation (see also Lentati et al. 2014) that allows for robust correction of DM variations during epochs without multifrequency data in the International Pulsar Timing Array (IPTA) data release (Verbiest et al. 2016). The DM noise model used by Lentati et al. (2016) assumes the power spectrum of the variations is in the form of a frequency-dependent power law. It also includes a term that provides power at a one-year period; any periodicity found would be in addition to that already included in the model. Lentati et al. (2016) do not find significant annual components in the data set, but this is not surprising as this effect is already being taken into account.

Lentati et al. (2017) find a covariance between temporal variations in scattering and measured DM. By analyzing PSR J1643-1224, identified here and in the literature as showing periodic variations in DM, Lentati et al. (2017) show that uncorrected temporal scattering variability introduces periodic structures in the DM time series. This effect is a possible cause of our measured variations for PSR J1643-1224 if the scattering is also variable on roughly annual timescales. We are not able to measure the scattering timescale for J1643-1224 in our nine-year data set due to insufficient frequency resolution, and significant variations in scattering timescales are not measurable for any pulsars (Levin et al. 2016). These variations could, however, contribute to annual DM variations for some pulsars. Future data releases using increased frequency resolution will incorporate scattering corrections (among others) and allow a comparison of the pre- and postcorrected DM time series.

The DM is highly anticorrelated with the solar elongation angle for PSRs J0030+0451 and J1614-2230, as can be seen in Table 5. There is a moderate correlation in PSRs J0023+0923 and J2010-1323. It is worth noting that in Figure 7, the DM measured for the second lowest solar elongation angle is higher than that for the minimum angle; this could be due to a random 

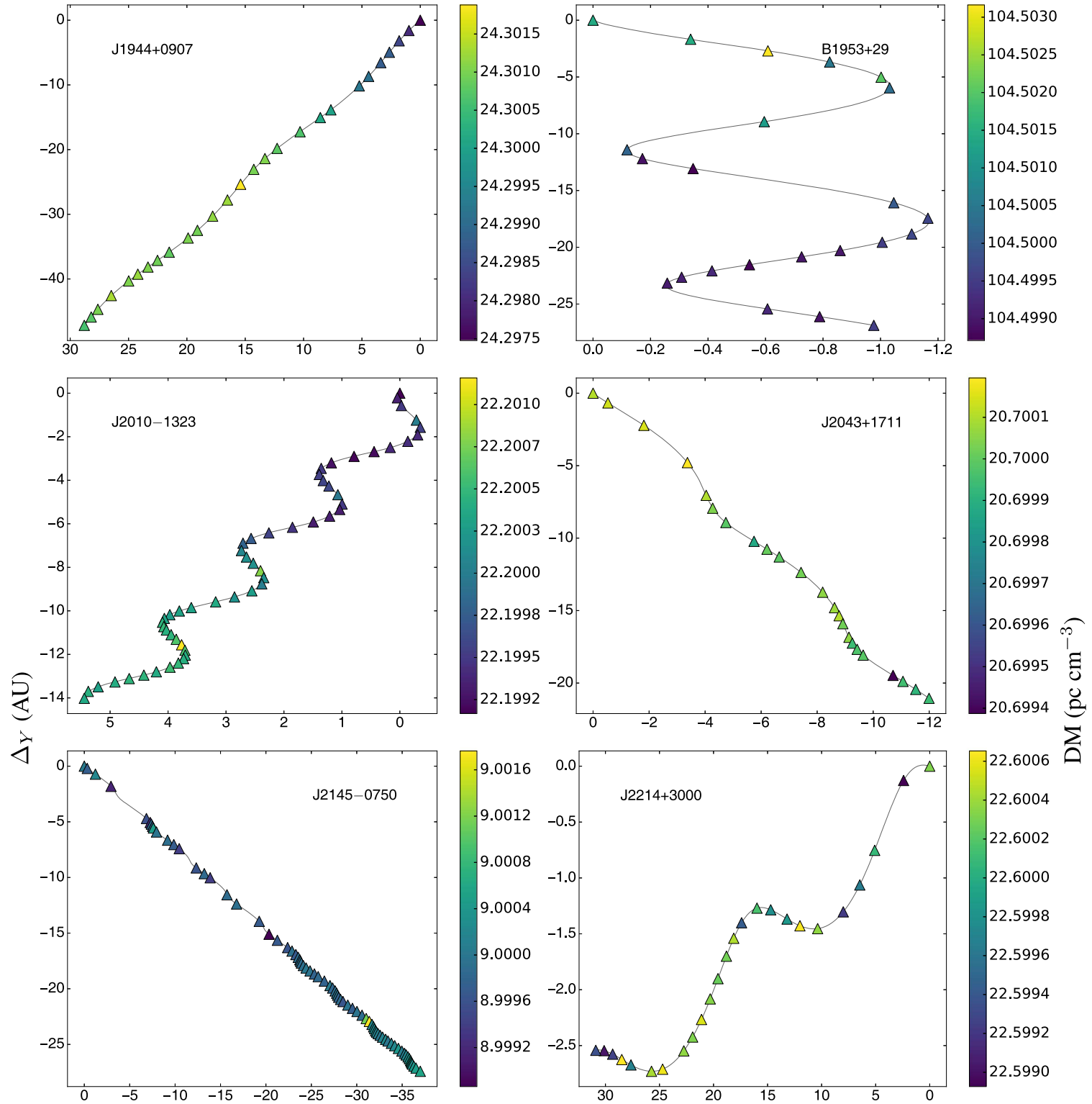

22.6006
22.6004
22.6002
22.6000
22.5998
22.5996
22.5994
22.5992
22.5990

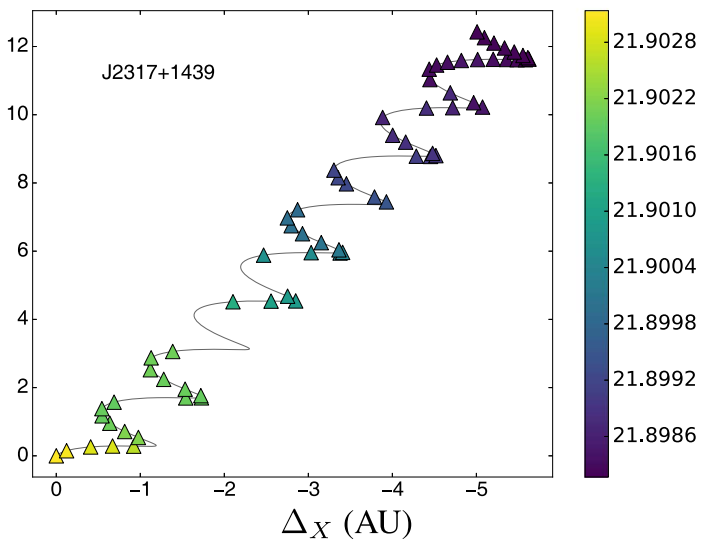

Figure 11. MSP trajectories are plotted with color mapping the DM at each epoch. The trajectories are calculated assuming that all the free electrons along the LOS are sitting in a phase screen halfway between the Earth and the MSP; the trajectory is then the projected motion of the pulsar on the phase screen. The axes depict the space traversed at the phase screen in au in the R.A. and decl. directions. The pulsar's motion starts at $(0,0)$. Pulsars closer to the ecliptic will show a tighter sinusoid than those farther away. The trajectory plot can be used to show limited localized structure. PSRs J1944+0907 and J2317+1439 show a monotonically increasing or decreasing DM, which could be due to a DM gradient along the direction of motion. PSR J2010-1323 shows an increasing DM in the direction of the pulsar's motion as well as high DM regions in the same part of its trajectory every year, suggesting a localized overdensity along the line of sight to the pulsar. 

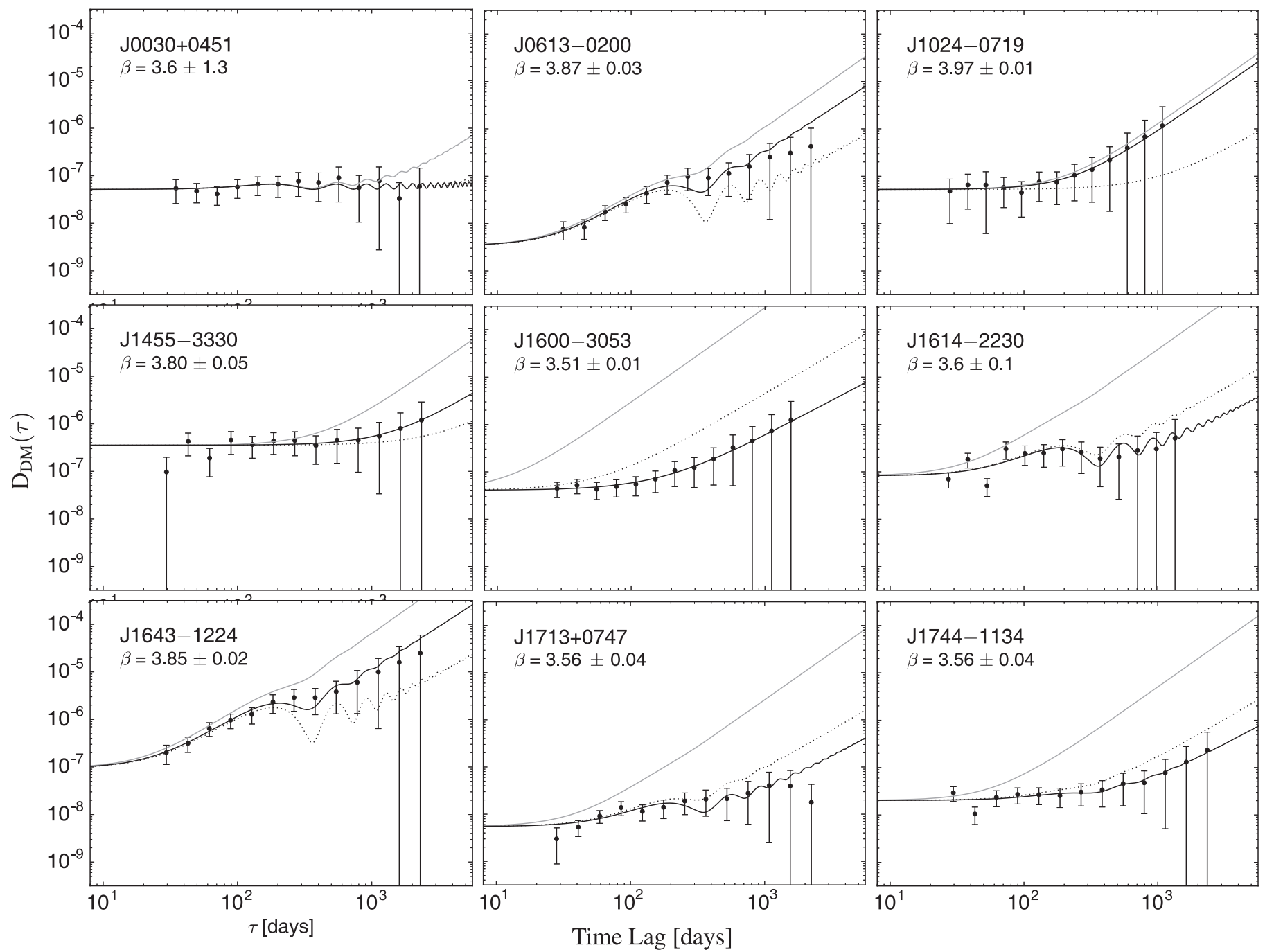

Figure 12. Structure functions for the DM variations, calculated for the MSPs with measured diffractive timescales. Error bars extending to the bottom of the frame signify an upper limit (in agreement with You et al. 2007). The solid gray lines signify a quadratic power law, and the dotted lines signify a Kolmogorov power law, which are anchored to the diffractive timescale, while the solid black lines are the best fits for the model. The errors associated with $\beta$ are $\pm 1 \sigma$ errors.

solar outflow that was not present during subsequent passes near the Sun. Comparison to an inverse square density model for the solar wind is difficult here as the model is an average and hence does not include solar events. The effects of the solar wind are clear in the pulsars with an apparent anticorrelation between the peak DM and solar elongation angle. However, because the solar wind can vary stochastically, the previous models may not represent the data fully.

The DM varies by one error bar over a timescale between one month and one year (30 days $<\delta t<365$ days) for 18 MSPs. An additional 11 MSPs had a $\delta t$ approximate to or smaller than the cadence of our observations (about one month). Only four MSPs showed no DM variations beyond measurement error on timescales less than one year, with no measurement calculable for four MSPs due to the absence of a predictable trend to use as a model. This illustrates how quickly the DM can vary by a significant amount and the necessity of observing at approximately week cadences and fitting for DM at every epoch.

The DM trajectory maps (Figures 8 through 11) are useful in visualizing various ISM features that the pulsar is encountering. A gradient in DM that is in the same direction as the pulsar's motion, as with PSRs J1024-0719 and J1643-1224, for example, could be indicative of an increasing or decreasing distance between Earth and the pulsar or of the pulsar moving through a density gradient. A gradient across the trajectory that is not in line with the pulsar's motion, as with PSRs J0613-0200 and J1614-2230, cannot arise solely from a change in distance. This coincides with the presence of systematic variations: pulsars with linear trends presenting as gradients along the direction of motion and periodic variations due to an overdensity that the pulsar repeatedly probes as it passes through the same LOS annually. Using PSR J0613-0200 as an example, assuming there is a $2 \mathrm{D}$ gradient in DM crossing the LOS in front of the pulsar, we find that the gradient has an amplitude of $d \mathrm{DM} / d \boldsymbol{x} \approx 2.6 \times 10^{-4} \mathrm{pc} \mathrm{cm}^{-3} \mathrm{au}^{-1}$ roughly perpendicular to the direction of motion on the sky.

\subsection{Structure Functions}

We have computed SFs for MSPs whose diffractive timescales could be calculated or obtained from the literature, as seen in Table 3. SFs (see Figures 12 and 13) could be calculated for 15 of 37 MSPs in the data release. Three MSPs (PSRs J1832-0836, B1953+29, and J2017+0603) whose diffractive timescales were available were omitted because there was fewer than two years of continuous DM 

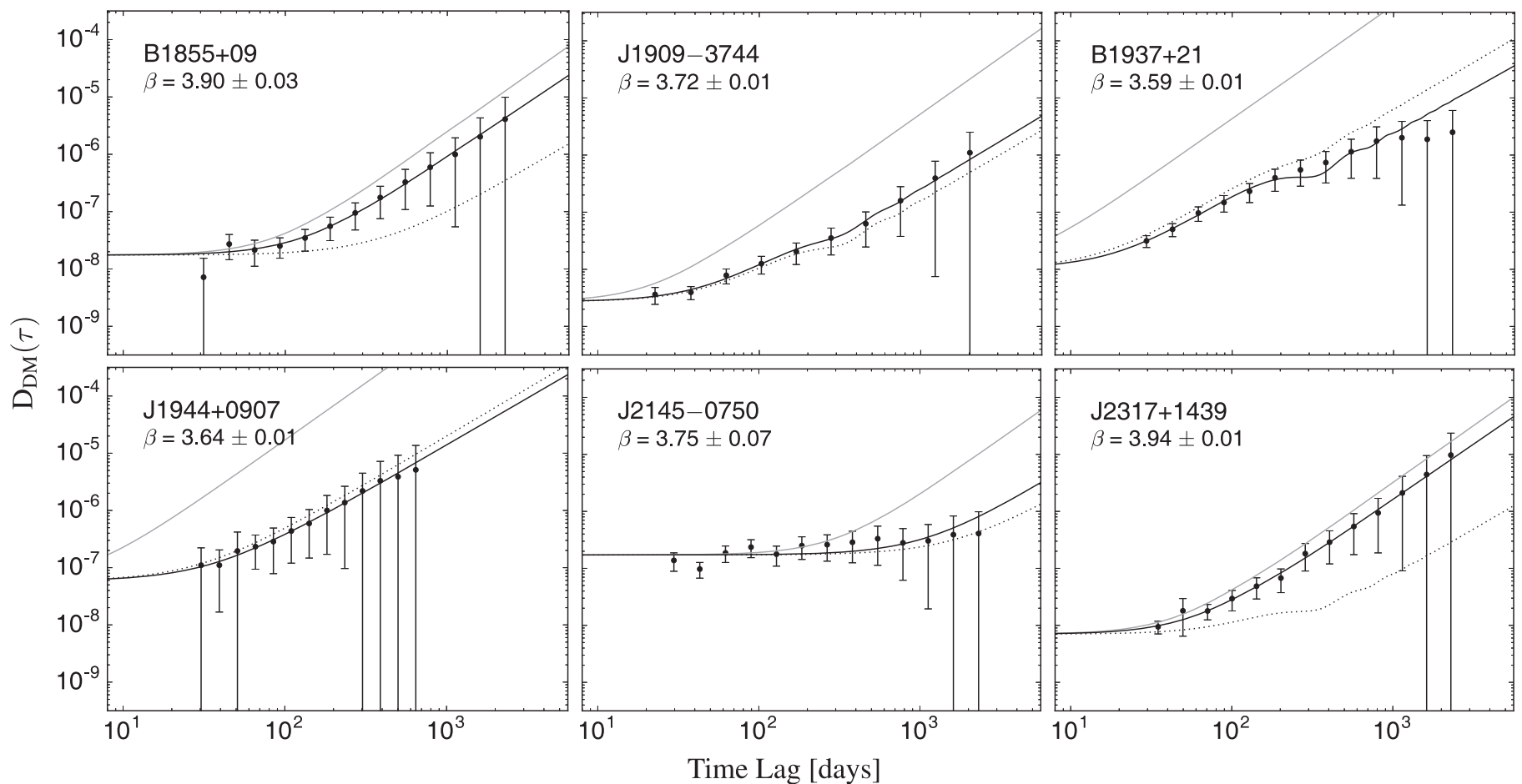

Figure 13. Structure functions for the DM variations, calculated for the MSPs with measured diffractive timescales. Error bars extending to the bottom of the frame signify an upper limit (in agreement with You et al. 2007). The solid gray lines signify a quadratic power law, and the dotted lines signify a Kolmogorov power law, which are anchored to the diffractive timescale, while the solid black lines are the best fits for the model. The errors associated with $\beta$ are $\pm 1 \sigma$ errors.

measurements. Fit power spectral values can also be seen in Table 3. PSRs J0030+0451 and J2145-0750 show fairly flat SFs, which is most likely a result of a data set dominated by white noise. Keith et al. (2013) show a SF for J2145-0750 exhibiting a similar structure at shorter time lags, but the error bars are too large to allow a detailed comparison.

For J1600-3053, Keith et al. (2013) measure a Kolmogorov SF; You et al. (2007) mention it as being quadratic at shorter time lags and becoming less steep at higher lags. Our analysis shows apparent white noise domination for time lags below $\sim 100$ days, beyond which the SF is bit more shallow than Kolmogorov for the majority of time lags.

PSRs J0613-0200, J1024-0719, J1643-1224, B1855+09, and $\mathrm{J} 2317+1439$ show nearly quadratic power spectra. Our calculated SF for J0613-0200 agrees with You et al. (2007). The SF for J1643-1224 resembles that in Keith et al. (2013). You et al. (2007) show a power-law exponent between the expected values for a quadratic and Kolmogorov medium at shorter time lags, with the SF exhibiting a power spectrum below Kolomogorov at higher lags. Our analysis shows a nearly quadratic power law with a distinct turnover present at a time lag of one year before climbing again at higher lags. This could be indicative of an underlying Kolmogorov medium.

You et al. (2007) do not calculate an SF for J1744-1134, but they predict it would be Kolmogorov based on previous $d \mathrm{DM} / d t$ measurements. Our calculation compares well to Keith et al. (2013), starting out dominated by white noise and then ending roughly Kolmogorov at higher lags. Our analysis finds a power spectrum below Kolmogorov.

PSRs J1614-2230, J2145-0750, J1909-3744, and J1944 +0907 are roughly Kolmogorov. PSR J1909-3744 compares well to the calculation in Lam et al. (2016). PSRs J1600-3053, $\mathrm{J} 1713+0747$, J1744-1134, and B1937+21 have power-law indices lower than expected for a Kolmogorov medium. PSR $\mathrm{J} 0030+0451$ has too large an uncertainty on $\beta$ for a definitive comparison.

The periods found by the SF fitting analysis agree within errors with those found by the DM time series trend analysis for all pulsars except one. A periodic trend was detected in the DM time series PSR B1855+09 but was not found by the SF analysis.

Here we discuss specific pulsars of interest.

\subsubsection{PSR $J 1713+0747$}

You et al. (2007) show the SF for J1713+0747 as being less steep than a quadratic power law at higher time lags, as do Keith et al. (2013). The SF calculated here looks almost white noise dominated; removing the DM event occurring around MJD 54750 (2008-2009) and recalculating the SF still yields what looks like a spectrum dominated by white noise. We do not believe this is purely white noise because of the correlated structure we see in the time series. However, if that power is evenly distributed over the range of time lags we are concerned with, then the SF will appear constant with lag.

Given the time of the observation immediately before the sharp decrease in DM on MJD 54751 and the epoch at which the DM is once again within $1 \sigma$ of the average value, we find an upper limit for the DM event of $\sim 200$ days; using the transverse velocity and distance from Table 1 and assuming the structure responsible is located at the pulsar give an upper limit size to the dispersing region of $4.0 \pm 0.1 \mathrm{au}$. With the increased amount of data in the IPTA data set, Lentati et al. (2016) are able to sample the DM event more effectively. They identify the DM event as spanning $\sim 100$ days, with the minimum DM occurring at $\sim$ MJD 54757. 

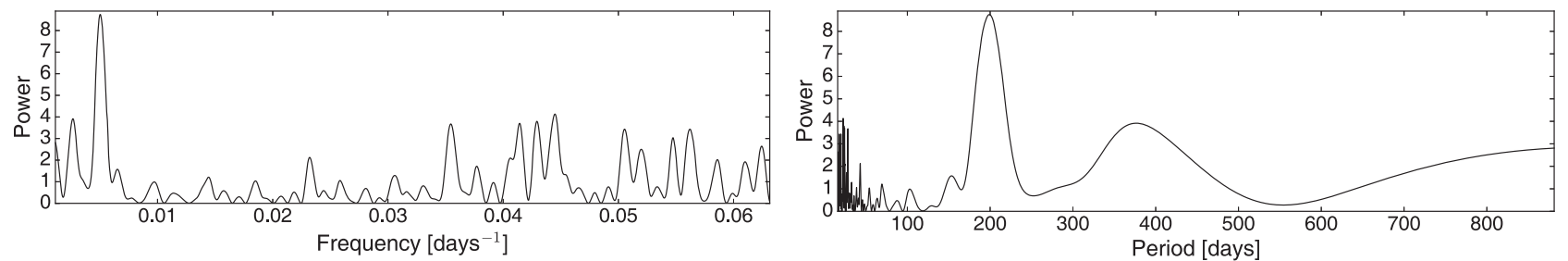

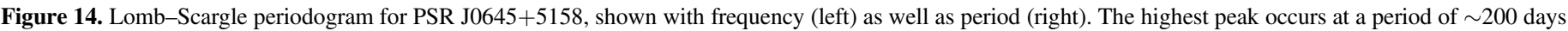

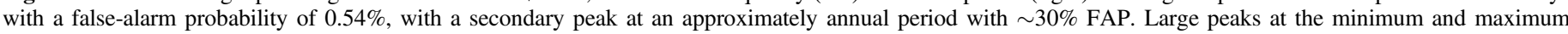
possible periods correspond to the cadence of observations and the length of the data set, respectively, and are therefore artifacts of the data acquisition.

\subsubsection{PSR B1855+09}

PSR B1855+09 shows a linear SF that does not align with the Kolmogorov or quadratic trend lines when using the diffractive timescale listed in Table 3 . This could be due to an incorrect or varying diffractive timescale; adding the diffractive timescale as another fitted parameter when fitting the SF gives a value of $\Delta \tau_{D}=55 \pm 1$ minutes, which is more than double the calculated values. The resulting SF is nearly quadratic, with some white noise dominating at small time lags.

\subsubsection{PSR B1937+21}

There is an extensive history of SF analysis for B1937+21. Kaspi et al. (1994) found a power-law exponent of $\beta=3.874 \pm 0.011$ with a little more than 8 years of data. Cordes et al. (1990) found a similar $\beta$ value that falls between a Kolmogorov and quadratic power spectrum, which agrees with our data at lower lags. Ramachandran et al. (2006) found a lower value of $\beta=3.66 \pm 0.04$ (compared to our $3.59 \pm 0.01$ ) from 1983 to 2004 that is consistent with a Kolmogorov medium. As with previous studies, Keith et al. (2013) also show a steady decrease in the DM through the end of 2010 and show a similar Kolmogorov-consistent SF. However, in 2011, the DM started to continuously increase through 2013, which is not a date range that any of the previously calculated SFs covered. It is likely that this latest increase in DM is the reason for the dips at higher lags that are not present in previous data sets.

\section{Discussion}

We examined a large set of DM time series from the NANOGrav data set and found evidence on timescales as small as weeks of variations in the vast majority of them. We found evidence of linear trends and annual periodicities, in addition to evidence for discrete ISM structures and one significant nonannual periodicity. We calculated and interpreted SFs for a subset of MSPs and examined DM variations due to LOS motions. These SFs often appear non-Kolmogorov, but we show that this can be due to the presence of trends in the data and does not necessarily indicate a non-Kolmogorov medium.

DM measurements can inform us about the free electron density along the LOS to a pulsar. In addition, trends due to the changing LOS over time aid in investigating structure in the ISM. Linear trends may be caused by parallel or transverse motion when the free electron density may be changing to a higher- or lower-than-average density in a region. Lam et al. (2016) show that DM variations due to a changing distance between Earth and the pulsar are dominated by parallel motion and that the transverse motion is negligible, entering only as a second-order consideration. The free electron density along a particular LOS is typically assumed to be temporally invariant.
Examining the scintillation parameters and flux densities of MSPs exhibiting linear DM trends can inform if this is an accurate assumption for that particular LOS. Looking at the LOS velocities for four pulsars, we expect that three of them (PSRs J1012+5307, J1024-0719, and J1738+0333) are consistent with exhibiting linear trends, due to the pulsar's increasing distance. The DM variation amplitude is much larger than expected given the average Galactic free electron density, indicating that the pulsars may be in overdense regions or ionize the materials along their paths (Chatterjee \& Cordes 2004). A transverse gradient may also contribute to the apparent DM variations; from our analysis we can only say that a changing distance may be ruled out as the cause. The velocity and direction of motion of PSR J1903+0327 suggest that its increasing distance from the Earth cannot explain its trend. Another effect (i.e., transverse component) must be present for this pulsar. Annual variations may be due to a variety of solar effects, and their amplitude is influenced by the relative velocity of the MSP when compared to the Earth's orbital motion as well as the Sun's velocity as it moves through the Galaxy. We see specific cases where the solar wind is the dominant factor in producing annual variations. For others, variations could be due to a cloud or gradient along the LOS. Scintillation parameters and fluxes could indicate whether variations were due to clumps, refraction, scattering variability, or local increases in electron density (such as solar wind; Stinebring \& Condon 1990; Clegg et al. 1998; Stinebring et al. 2000; Lentati et al. 2017). Future work may result in some determination, particularly if scintillation parameters over time are available and incorporated.

The relationship between linear and periodic variations and the direction of a gradient along the LOS can also be visualized by DM trajectory maps. Five MSPs show only annual trends and 14 show only linear trends, while 14 exhibit both trends. More than one-half of the MSPs showed significant DM variation beyond our measurement error over the timescale of one month to one year. Eleven MSPs have timescales less than 31 days, which is on par with the average cadence of our observations. Of those, four MSPs have timescales of 14 days or less, which is the size of the fitting window used for DMX in the nine-year data release. It is therefore imperative that we fit for DM at every epoch because of the scale of the variations over these timescales, as well as observe as often as possible to minimize the time between DM measurements because of the rapid variation seen in some MSPs.

For three PSRs, the SFs appear to be dominated by white noise, resulting in a flat power spectrum. Three MSPs have very nearly quadratic power spectra, with two (PSRs $\mathrm{J} 1024-0719$ and $\mathrm{J} 2317+1439$ ) having a $\beta$ value within 1\% of quadratic. Lam et al. (2016) suggest that discrete structures in the ISM as well as the changing distance will contaminate 
the SF, resulting in a quadratic power spectrum. This steeperthan-Kolmogorov value could indicate that the time lag is smaller than the crossing time for the structure probed during the time series. Values higher than consistent with a Kolmogorov medium could be attributed to present trends or systematic variations in addition to a Kolmogorov medium, particularly with the previously discussed difficulties in disentangling a linear trend component from a stochastic one. We cannot impose priors without assuming something about the contributions from the ISM that we are trying to constrain, and which may not actually be the case. There are also more possible sources of error in the calculation of the $\beta$ values than we have included. We have accounted for the random and stochastic uncertainties but not systematic uncertainties, which can result from variability of white noise statistics over time from changing back ends, the variation in the diffractive timescale, and the fact that the models used here could be incomplete in describing the ISM. Therefore, while the $\beta$ values presented here are illustrative, their errors bars are likely underestimated. In addition, care should be taken when using these values to make inferences about the ISM because possible covariances and systematics may be present.

The NANOGrav project received support from the National Science Foundation (NSF) PIRE program award number 0968296 and NSF Physics Frontier Center award number 1430284. M.L.J. acknowledges support from West Virginia University through the STEM Mountains of Excellence Fellowship. NANOGrav research at UBC is supported by an NSERC Discovery Grant and Discovery Accelerator Supplement and the Canadian Institute for Advanced Research. J.A.E. acknowledges support by NASA through Einstein Fellowship grant PF3-140116. Portions of this research were carried out at the Jet Propulsion Laboratory, California Institute of Technology, under a contract with the National Aeronautics and Space Administration. T.T.P. was a student at the National Radio Astronomy Observatory (NRAO) while this project was undertaken. Data for the project were collected using the facilities of the NRAO and the Arecibo Observatory. The NRAO is a facility of the NSF operated under cooperative agreement by Associated Universities, Inc. The Arecibo Observatory is operated by SRI International under a cooperative agreement with the NSF (AST-1100968), and in alliance with the Ana G. Méndez-Universidad Metropolitana, and the Universities Space Research Association.

Author contributions. M.L.J. carried out the analysis and prepared the majority of the text, figures, and tables. M.A.M., M.T.L., J.M.C., L.L., and S.C. helped with the development of the framework and text. Z.A., K.C., P.B.D., T.D., J.A.E., R.D.F., E.F., M.E.G., G.J., M.L.J., M.T.L., L.L., M.A.M., D.J.N., T.T.P., S.M.R., I.H.S., K.S., J.K.S., and W.W.Z. all ran observations and developed timing models for the NG9 data set; additional specific contributions are outlined in Arzoumanian et al. (2015).

\section{References}

Andrae, R., Schulze-Hartung, T., \& Melchior, P. 2010, arXiv:1012.3754 Antoniadis, J. I. 2013, PhDT thesis, Univ. Bonn

Arzoumanian, Z., Brazier, A., Burke-Spolaor, S., et al. 2015, ApJ, 813, 65

Chatterjee, S., \& Cordes, J. M. 2004, ApJL, 600, L51

Clegg, A. W., Fey, A. L., \& Lazio, T. J. W. 1998, ApJ, 496, 253

Cordes, J. M., \& Lazio, T. J. W. 2002, arXiv:astro-ph/0207156

Cordes, J. M., \& Shannon, R. M. 2010, arXiv:1010.3785

Cordes, J. M., Shannon, R. M., \& Stinebring, D. R. 2016, ApJ, 817, 16

Cordes, J. M., Wolszczan, A., Dewey, R. J., Blaskiewicz, M., \& Stinebring, D. R. 1990, ApJ, 349, 245

Demorest, P. B., Ferdman, R. D., Gonzalez, M. E., et al. 2013, ApJ, 762, 94

Fonseca, E., Stairs, I. H., \& Thorsett, S. E. 2014, ApJ, 787, 82

Frisch, P. C., Redfield, S., \& Slavin, J. D. 2011, ARA\&A, 49, 237

Hamilton, P. A., Hall, P. J., \& Costa, M. E. 1985, MNRAS, 214, 5P

Isaacman, R., \& Rankin, J. M. 1977, ApJ, 214, 214

Jenet, F. A., Hobbs, G. B., Lee, K. J., \& Manchester, R. N. 2005, ApJL, 625, L123

Johnston, S., Nicastro, L., \& Koribalski, B. 1998, MNRAS, 297, 108

Kaplan, D. L., Kupfer, T., Nice, D. J., et al. 2016, ApJ, 826, 86

Kaspi, V. M., Taylor, J. H., \& Ryba, M. F. 1994, ApJ, 428, 713

Keith, M. J., Coles, W., Shannon, R. M., et al. 2013, MNRAS, 429, 2161

Lam, M. T., Cordes, J. M., Chatterjee, S., et al. 2016, ApJ, 821, 66

Lam, M. T., Cordes, J. M., Chatterjee, S., \& Dolch, T. 2015, ApJ, 801, 130

Lentati, L., Alexander, P., Hobson, M. P., et al. 2014, MNRAS, 437, 3004

Lentati, L., Kerr, M., Dai, S., et al. 2017, MNRAS, 468, 1474

Lentati, L., Shannon, R. M., Coles, W. A., et al. 2016, MNRAS, 458, 2161

Levin, L., McLaughlin, M. A., Jones, G., et al. 2016, ApJ, 818, 166

Manchester, R. N., Hobbs, G. B., Teoh, A., \& Hobbs, M. 2005, AJ, 129, 1993

Matthews, A. M., Nice, D. J., Fonseca, E., et al. 2016, ApJ, 818, 92

McLaughlin, M. A. 2013, CQGra, 30, 224008

Nicastro, L., Nigro, F., D’Amico, N., Lumiella, V., \& Johnston, S. 2001, A\&A, 368, 1055

Petroff, E., Keith, M. J., Johnston, S., van Straten, W., \& Shannon, R. M. 2013, MNRAS, 435, 1610

Ramachandran, R., Demorest, P., Backer, D. C., Cognard, I., \& Lommen, A. 2006, ApJ, 645, 303

Rankin, J. M., \& Roberts, J. A. 1971, in IAU Symp. 46, The Crab Nebula, ed. R. D. Davies \& F. Graham-Smith (Dordrecht: Reidel), 114

Reardon, D. J., Hobbs, G., Coles, W., et al. 2016, MNRAS, 455, 1751

Rickett, B. J. 1990, ARA\&A, 28, 561

Scargle, J. D. 1982, ApJ, 263, 835

Siemens, X., Ellis, J., Jenet, F., \& Romano, J. D. 2013, CQGra, 30, 224015

Stinebring, D. R., \& Condon, J. J. 1990, ApJ, 352, 207

Stinebring, D. R., Smirnova, T. V., Hankins, T. H., et al. 2000, ApJ, 539, 300

Verbiest, J. P. W., Lentati, L., Hobbs, G., et al. 2016, MNRAS, 458, 1267

Vigeland, S. J., \& Siemens, X. 2016, PhRvD, 94, 123003

You, X. P., Hobbs, G. B., Coles, W. A., et al. 2007, MNRAS, 378, 493 